\title{
Multi-wavelength fine structure and mass flows in solar microflares ${ }^{\star}$
}

\author{
S. Berkebile-Stoiser ${ }^{1}$, P. Gömöry ${ }^{1,2}$, A. M. Veronig ${ }^{1}$, J. Rybák ${ }^{2}$, and P. Sütterlin ${ }^{3}$ \\ 1 Institute of Physics, University of Graz, Universitätsplatz 5, 8010 Graz, Austria \\ e-mail: sigrid.berkebile-stoiser@uni-graz.at \\ 2 Astronomical Institute, Slovak Academy of Sciences, 05960 Tatranská Lomnica, Slovakia \\ 3 Institute for Solar Physics, The Royal Swedish Academy of Sciences, Alba Nova University Center, 10691 Stockholm, Sweden
}

Received 18 March 2009 / Accepted 30 July 2009

\begin{abstract}
Aims. We study the multi-wavelength characteristics at high spatial resolution, as well as chromospheric evaporation signatures of solar microflares. To this end, we analyze the fine structure and mass flow dynamics in the chromosphere, transition region and corona of three homologous microflares (GOES class <A9/0.7 with/without background), which occurred on July 4, 2006 in AR 10898. Methods. A multi-wavelength analysis using temporally and spatially highly resolved imaging data from the Dutch open telescope $(\mathrm{H} \alpha, \mathrm{Ca}$ II H$)$, the transition region and coronal explorer $(17.1 \mathrm{~nm})$, the extreme-ultraviolet imaging telescope $(19.5 \mathrm{~nm})$, and the Reuven Ramaty high energy solar spectroscopic imager $(~ \gtrsim 3 \mathrm{keV})$ was carried out. EUV line spectra provided by the coronal diagnostic spectrometer are searched for Doppler shifts in order to study associated plasma flows at chromospheric (He I, $T \sim 3.9 \times 10^{4} \mathrm{~K}$ ), transition region (e.g. O V, $T \sim 2.6 \times 10^{5} \mathrm{~K}$ ), and coronal temperatures (Si XII, $T \sim 2 \times 10^{6} \mathrm{~K}$ ). RHESSI X-ray spectra provide information about non-thermal electrons.

Results. The multi-wavelength appearance of the microflares is in basic agreement with the characteristics of large flares. For the first event, a complex flare sequence is observed in TRACE $17.1 \mathrm{~nm}$ images $(T \approx 1 \mathrm{MK})$, which show several brightenings, narrow loops of enhanced emission, and an EUV jet. EIT $19.5 \mathrm{~nm}$ data $(T \approx 1.5 \mathrm{MK})$ exhibit similar features for the third event. DOT measurements show finely structured chromospheric flare brightenings for all three events, loop-shaped fibrils of increased emission between $\mathrm{H} \alpha$ brightenings, as well as a similar feature in Ca II. For all three events, a RHESSI X-ray source (3-8 keV, $T \gtrsim 10 \mathrm{MK})$ is located in between two chromospheric brightenings situated in magnetic flux of opposite polarity. We find the flow dynamics associated with the events to be very complex. In the chromosphere and transition region, CDS observed downflows for the first $\left(v \lesssim 40 \mathrm{~km} \mathrm{~s}^{-1}\right)$, and upflows for the second event $\left(v \lesssim 40 \mathrm{~km} \mathrm{~s}^{-1}\right)$. During the third microflare, we find upflows of $\lesssim 20 \mathrm{~km} \mathrm{~s}^{-1}$ and also weak downflows of $\$ 20 \mathrm{~km} \mathrm{~s}^{-1}$ in two separate brightenings. For all three microflares, multi-component fitting is needed for several profiles of He I, O v, and $\mathrm{Ne}$ VI lines observed at the flare peaks, which indicate spatially unresolved, oppositely directed flows of $\lesssim 180 \mathrm{~km} \mathrm{~s}^{-1}$. We interpret these flows as twisting motions of the flare loops. Loop-shaped fibrils in between $\mathrm{H} \alpha$ brightenings showing opposite flow directions $\left(v \approx 5 \mathrm{~km} \mathrm{~s}^{-1}\right)$ are also observed in DOT H $\alpha$ Dopplergrams. RHESSI X-ray spectra show evidence of non-thermal bremsstrahlung for two of the three microflares. The electron beam flux density deposited in the chromosphere for these events is estimated to straddle the threshold heating flux between gentle and explosive evaporation.
\end{abstract}

Key words. Sun: flares - Sun: chromosphere - Sun: corona

\section{Introduction}

Solar flares are believed to be powered by magnetic reconnection in the corona where the magnetic energy released is to a great part used up for energizing particles beyond the thermal range. Non-thermal electrons travel towards the solar surface and finally encounter dense plasma in the transition region and chromosphere where they deposit the bulk of their energy via Coulomb collisions with the ambience. Because of the strong energy input the chromospheric plasma is heated to temperatures $\gtrsim 10^{7} \mathrm{~K}$ and expands upwards into the corona. This process was termed "chromospheric evaporation" (see Sturrock 1973). According to hydrodynamic simulations, hot upflows reaching supersonic velocities of several hundred $\mathrm{km} \mathrm{s}^{-1}$ should be detectable in the impulsive phase of solar flares (see Fisher et al. 1985a,b,c; Abbett \& Hawley 1999; Allred et al. 2005). A downward push of the lower chromosphere is also expected during

* Appendix A and the movie are only available in electronic form at http://www. aanda.org times of large energy input into the chromosphere, which establishes momentum balance with the hot upflows (Fisher 1989). Whereas this sequence called "explosive evaporation" is suggested for flares that deposit high non-thermal electron flux density in the chromosphere, simulations also suggest that evaporation can be "gentle" for flares with lower electron flux density. For gentle evaporation, the chromosphere is expected to slowly expand upwards at several tens of $\mathrm{km} \mathrm{s}^{-1}$ to adjust to a new equilibrium position.

Upflows of hot plasma were indeed found in flares observed in soft X-ray emission lines by the bent crystal spectrometer on board the solar maximum mission (SMM/BCS; Acton et al. 1980) and the Bragg crystal spectrometer on the Yohkoh satellite (Culhane et al. 1991). In flares observed with these instruments, strongly blueshifted lines appeared that indicated upflow velocities $\gtrsim 100 \mathrm{~km} \mathrm{~s}^{-1}$ (e.g. Doschek et al. 1980; Feldman et al. 1980; Antonucci et al. 1982; Fludra et al. 1989; Antonucci et al. 1990; Mariska et al. 1993; Brosius \& Phillips 2004). As these observations were disk-integrated and did not feature any spatial 
resolution, a direct comparison between flare kernels and the position where the plasma flows originated was impossible. The coronal diagnostic spectrometer (CDS; Harrison et al. 1995) on the solar and heliospheric observatory (SOHO; Domingo et al. 1995), on the other hand, allows us to study flows at chromospheric to coronal temperatures at excellent time and spatial resolution.

Although CDS lacks the possibility studying the hottest part of the flare plasma, it has been successfully used for a couple of flare studies which aimed at deriving the characteristics of evaporation flows during flares. Of special importance in this context are observations where the slit is situated directly at the flare energy deposit site. In one of the first CDS flare studies, Czaykowska et al. (1999) find downflows at the end of bright post flare loops but also strong, hot upflows at their outer edge even in the late phase of an M class flare. Brosius (2003) also finds upflows in the impulsive phase and "warm rain" due to cooling flare plasma in an important flare. The observed flow velocities in comparison with predictions of hydrodynamic simulations were studied by Brosius \& Phillips (2004), who report observations of a flare jointly observed by CDS and Yohkoh/BCS suggesting momentum balance between up- and downward flowing material in the impulsive phase. Teriaca et al. (2006) find an order of magnitude agreement of the momenta of cospatial, oppositely directed flows in a small two ribbon flare. The combination of flare X-ray spectra and images and CDS Doppler velocities enabled Milligan et al. (2006) to demonstrate observations in rough agreement with simulations of explosive chromospheric evaporation.

At the lower end of the energy distribution of solar flares range micro- and nanoflares which are named after the fraction of thermal plasma energy they contain compared with the largest observed events (Aschwanden 2004). In the micro- and nanoflare coronal heating model, a multitude of such small flares are proposed to supply the energy to heat the solar corona to the observed temperatures of $\sim 1-3$ MK (e.g. Parker 1988). Additionally to heating plasma, there also has to be a mechanism to constantly transport matter into the corona which would otherwise disappear due to solar wind flows. This could in principle be achieved by chromospheric evaporation associated with micro- and nanoflares (Aschwanden et al. 2007; Stoiser et al. 2008). Observations show that microflares behave very similar to large flares with respect to their multi-wavelength and X-ray spectral properties (Krucker et al. 2002; Liu et al. 2004; Kundu et al. 2006; Stoiser et al. 2007). This suggests that the same dynamic processes like chromospheric evaporation due to fast electron beams also occur in microflares. However, evidence of mass flows and their characteristics like the speed and flow direction in different parts of the solar atmosphere for microflares is extremely rare. Brosius \& Holman (2009) studied a compact RHESSI microflare (GOES class A5) and found upflows $\lesssim 20 \mathrm{~km} \mathrm{~s}^{-1}$ in chromospheric and coronal lines which are consistent with gentle evaporation. Analyzing small C-class flares, Falchi et al. (2006) and Milligan (2008) report evidence for conductive evaporation and direct heating to create hot, coronal flare plasma instead of beam driven chromospheric evaporation. As mainly observations of strong "microflares" (GOES C) have been reported, the basic properties of plasma flows in the smallest microflares compared with the theoretically expected behavior as outlined by flare simulations still needs to be established. Also, the amount of plasma transported into the corona due to evaporation flows remains unclear.

In this paper, we report on 3 microflares (GOES class $<$ A9/A1 with/without background) for which imaging and spectroscopic observations of the chromosphere, transition region and corona with high temporal and spatial resolution were achieved. As far as we are aware, these are the smallest flare events so far studied for chromospheric evaporation flows. Imaging of the chromosphere at a resolution as high as $0.2^{\prime \prime}$ by the Dutch open telescope as well as images of the transition region and corona provided by the transition region and coronal explorer (resolution of $1^{\prime \prime}$ ) reveal the fine structure of the microflares in the entire solar atmosphere. The analysis of EUV line spectra recorded directly at the flare sites by CDS allows us to identify plasma flows in the chromosphere, transition region and corona. The energy input into the chromosphere by nonthermal electrons is studied with RHESSI X-ray spectra. With our data set, we are able to give a detailed picture of the processes in selected microflares. Such comprehensive case studies are important in the frame of the micro-and nanoflare coronal heating model as well as for the understanding of the flare process in general.

\section{Instrument description and data reduction}

\subsection{Observing campaign and ground based instruments}

In this paper, we present data acquired during a coordinated observing campaign performed in the period June 28-July 12, 2006 which aimed to explore the dynamics of microflares and the chromospheric network. The SOHO joint observing program JOP 171 included the operation of selected instruments onboard SOHO and the transition region and coronal explorer (TRACE; Handy et al. 1999). The space instruments were supported by the ground-based Dutch open telescope (DOT; Hammerschlag $\&$ Bettonvil 1998). DOT provided tomographic (nearly simultaneous) filtergrams of the Sun's photosphere and chromosphere in the blue and red continuum (432 and $651 \mathrm{~nm}$ ), the $\mathrm{G}$ Band (430.5 nm), the $\mathrm{Ca}$ II $\mathrm{H}$ line $(396.8 \mathrm{~nm})$ and the $\mathrm{H} \alpha$ line $(656.3 \mathrm{~nm}, \pm 0.35 \AA)$. Speckle reconstruction of the data served to achieve the telescope diffraction limit of $0.2^{\prime \prime}$ for a subfield sized from $72^{\prime \prime} \times 60^{\prime \prime}$ to $92^{\prime \prime} \times 75^{\prime \prime}$. The spatial resolution of $0.2^{\prime \prime}$ applies to images recorded in the $\mathrm{G}$ Band whereas the resolution is already slightly lower for $\mathrm{H} \alpha$ filtergrams $\left(0.3^{\prime \prime}\right)$. The time cadence of the speckeled images used here was better than $26 \mathrm{~s}$. DOT Doppler maps were reconstructed from the $\mathrm{H} \alpha$ wing intensities. For each pixel, the difference of normalized intensities observed in the DOT passbands set in the $\mathrm{H} \alpha$ red and blue wing $\left(\left[I_{\mathrm{b}}-I_{\mathrm{r}}\right] /\left[I_{\mathrm{b}}+I_{\mathrm{r}}\right]\right)$ was used as a proxy for flow speeds. Details on the telescope, the DOT tomographic multi-wavelength imaging and data acquisition system, speckle reconstruction and reduction procedures are provided in Sütterlin et al. (2001) and Rutten et al. (2004).

\subsection{Space-borne instruments}

Space-borne instruments coordinated with the DOT during JOP 171 were SOHO/CDS, the Michelson Doppler imager (SOHO/MDI; Scherrer et al. 1995) and TRACE. For our study we also use data of the extreme-ultraviolet imaging telescope (SOHO/EIT; Delaboudinière et al. 1995) and the Reuven Ramaty high energy solar spectroscopic imager (RHESSI; Lin et al. 2002).

The normal incidence spectrometer (NIS) on CDS (Harrison et al. 1995) provided simultaneous EUV line spectra of six chromospheric, transition region and coronal lines which were used to determine line of sight flow speeds in the observed microflare kernels. We selected emission lines of the following 
ions, and give their nominal wavelength and formation temperature: He I $\left(58.43 \mathrm{~nm}, T \sim 3.9 \times 10^{4} \mathrm{~K}\right)$, O III $(59.96 \mathrm{~nm}$, $\left.T \sim 1.2 \times 10^{5} \mathrm{~K}\right), \mathrm{O}$ V $\left(62.97 \mathrm{~nm}, T \sim 2.6 \times 10^{5} \mathrm{~K}\right), \mathrm{Ne} \mathrm{VI}$ $\left(56.28 \mathrm{~nm}, T \sim 4.2 \times 10^{5} \mathrm{~K}\right), \mathrm{Mg} \mathrm{IX}\left(36.8 \mathrm{~nm}, T \sim 1.0 \times 10^{6} \mathrm{~K}\right)$ and Si XII $\left(52.07 \mathrm{~nm}, T \sim 2 \times 10^{6} \mathrm{~K}\right)$. The CDS slit $\left(2^{\prime \prime} \times 140^{\prime \prime}\right.$, pixel size $\left.2^{\prime \prime} \times 1.6^{\prime \prime}\right)$ was set fixed in a sit-and-stare mode for $\sim 5.45 \mathrm{~h}$ acquiring observations at a cadence of $15 \mathrm{~s}$ (10 s exposure plus $\sim 5 \mathrm{~s}$ read-out time). Four raster scans, i.e. 20 successive steps of the slit in $x$-direction corresponding to a $40^{\prime \prime} \times 140^{\prime \prime}$ map were obtained before/after the 1D observing sequence. These rasters were used for the co-alignment of the CDS data with the other data sets. Raw 1D and 2D data were corrected for the CCD bias, deviations in exposure time, flat-field and cosmic ray hits by standard calibration software available in the solar software tree $(\mathrm{SSW})$.

As the wavelength axis of CDS is not absolute it has to be calibrated before Doppler shifts to the wavelength at rest can be determined. For this purpose, a mean line profile was calculated from the pixels in the nearest raster image. Pixels with intensities above a threshold assumed to constitute the highest emission coming from the quiet sun were excluded. Thus, bright features like sunspot plumes and their associated flows do not influence the wavelength calibration (see e.g. Maltby et al. 1999). The thus obtained mean profile was supposed to be unshifted, i.e. all up- and downflows in the image were assumed to balance each other. The wavelength of this profile was set to the laboratory wavelength of the considered line according to MacPherson \& Jordan (1999) corrected by the deviations suggested by Peter \& Judge (1999). These corrections account for the constantly observed transition region and coronal blue and redshifts in the solar atmosphere (see Gömöry et al. 2006). The largest correction was applied to the $\mathrm{O} v$ line for which a constant redshift of $\sim 10 \mathrm{~km} \mathrm{~s}^{-1}$ is assumed, whereas the chromospheric He I line is presented to be close to unshifted by Peter \& Judge (1999). For all other lines, the correction into the red or blue are $\lesssim 6 \mathrm{~km} \mathrm{~s}^{-1}$. We estimate the error in the velocity calibration to range within $\pm 10 \mathrm{~km} \mathrm{~s}^{-1}$. The calibrated line spectra were fitted with a single broadened Gaussian ${ }^{1}$. Alternatively, as several profiles at the 3 events' peaks were clearly composed of more than one component, we applied a two component Gaussian fit in these cases. The line fits provided the intensities, wavelength positions of the core and the width of the line. Of major interest for our analysis are the Doppler velocities which were calculated from the wavelength shift of the line centroid relative to its nominal wavelength.

For our campaign, MDI white light maps and high resolution line of sight magnetograms at a pixel resolution of $\sim 0.6^{\prime \prime}$ and a time cadence of $1 \mathrm{~min}$ were requested. Unfortunately, this observing mode ceased to operate $\sim 45 \mathrm{~min}$ before the first microflare in the present study occurred. Thus, a high resolution magnetogram corrected for solar differential rotation was used to study the magnetic context.

We also found one image showing one of our events in the EIT $19.5 \mathrm{~nm}$ line (Fe XII). EIT observed on this day at a time cadence of $\sim 12 \mathrm{~min}$ and at a pixel resolution of $\sim 2.6^{\prime \prime}$. The dark current and flat field were corrected with SSW routines prepared for EIT data analysis.

The TRACE satellite provided image sequences in the $17.1 \mathrm{~nm}$ passband showing plasma at a temperature of $\sim 10^{6} \mathrm{~K}$.

\footnotetext{
1 The broadended Gaussian profile is used for CDS observations acquired after the recovery of the SOHO spacecraft in 1998. Details can be found in CDS software note no. 53 at http://orpheus. nascom. nasa.gov/cds/swnote/cds_swnote_53.ps
}

The filtergrams feature a high spatial resolution of $0.5^{\prime \prime} /$ pixel for a $\sim 511^{\prime \prime} \times 511^{\prime \prime}$ field of view (FoV) and a time cadence of $\sim 90 \mathrm{~s}$. The TRACE images were flat-fielded and the dark current and cosmic ray hits were removed by using standard SSW routines.

For identifying microflares, we used observations of the RHESSI instrument which observes flare X-ray and $\gamma$-ray emission $\gtrsim 3 \mathrm{keV}$ for a full sun field of view. RHESSI is capable of high resolution spectroscopy and imaging. At energies $\lesssim 100 \mathrm{keV}$, RHESSI features excellent spatial (down to $\sim 2.3^{\prime \prime}$ ) and spectral resolution $(\sim 1 \mathrm{keV})$. Most importantly for our study, the RHESSI germanium detectors are extraordinarily sensitive (Smith et al. 2002) and are able to observe small microflares not detected by the GOES (geostationary operational environmental satellites) X-ray detector used for flare classification. The analysis of RHESSI data was carried out with SSW software. Images were always reconstructed with the Pixon algorithm (Hurford et al. 2002) and spectral fitting was done utilizing the OSPEX package (Schwartz et al. 2002). The RHESSI detectors were not attenuated during the microevents which ensures highest sensitivity.

\subsection{Data co-alignment}

A lot of work was invested in order to carefully co-align this extensive data set. The coordinate shift between different instruments was derived by the 2-dimensional cross-correlation of images or CDS raster scans taken in similar temperature ranges and recorded close in time to each other. The reference coordinate system for our data set was EIT. First, CDS He I $58.4 \mathrm{~nm}$ raster images were coaligned with the EIT He II $30.4 \mathrm{~nm}$ full disk image closest in time. TRACE $17.1 \mathrm{~nm}$ maps data were aligned with the EIT maps in the same wavelength band, and the pointing information was used to update all other TRACE filtergrams, thereby also accounting for the varying field of view of the individual TRACE filters. The co-aligned TRACE white light images were the reference for the MDI high resolution white light and magnetogram maps. Images acquired in the DOT Ca II H channel were co-aligned with TRACE $160 \mathrm{~nm}$ images and served as reference for the remaining DOT channels. As DOT tracked the major sunspot in its FoV during the observing run, the cross-correlation of DOT data with TRACE images accounted not only for translation in $x$ - and $y$-direction (resolution of $0.5^{\prime \prime}$ ), but also for the image tilt (resolution of $\sim 0.1 \mathrm{deg}$ ). The errors to be expected in the obtained co-alignment were estimated by repeating the procedure and choosing a different order of instruments/filters. The thus derived uncertainties for all instruments are $\lesssim 1.2^{\prime \prime}$. For RHESSI images, we did not apply a change of the coordinate system but used the reconstructed images directly as the RHESSI pointing is accurate to $<1$ " (Lin et al. 2002).

\section{Microflare fine structure}

On July 4, 2006, the flare activity was low with 27 flares of class A9.9-C1.2 stated in the GOES event summary. The RHESSI flare list (not corrected for satellite night times) recorded 19 events emitting in the $3-6 \mathrm{keV}$ range and 2 events with emission up to $12-25 \mathrm{keV}$.

The instruments included in our campaign were pointed to AR 10898 located $200^{\prime \prime}$ from disk center. An overview on the data set illustrating the appearance of AR 10898 in TRACE white light and EUV images, in selected DOT channels and an MDI magnetogram can be seen in Fig. 1. The active region consisted of a major sunspot (Mt. Wilson class $\beta$ ) which was 

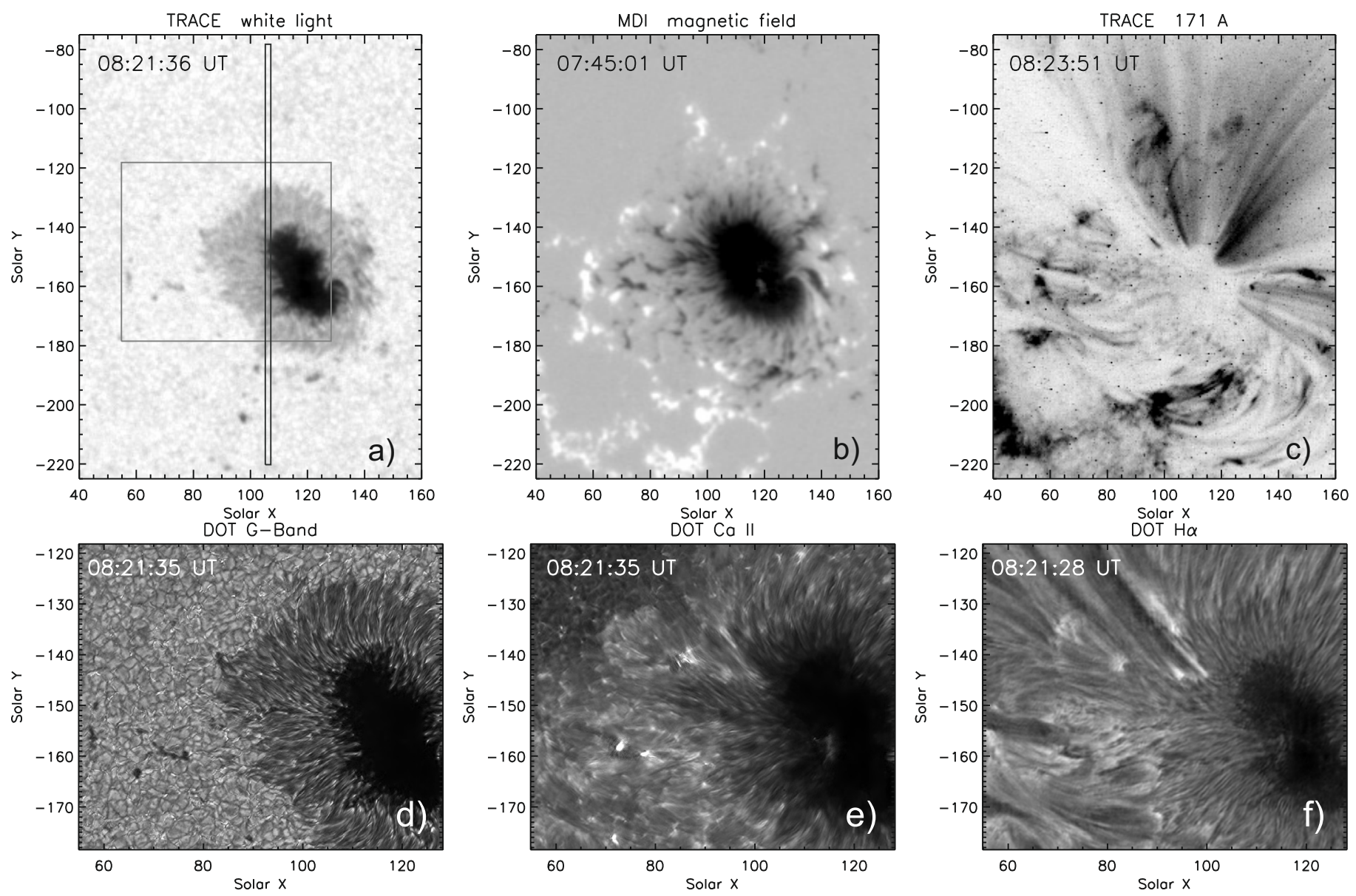

Fig. 1. AR 10898 as observed by TRACE, MDI and DOT before the 08:26 UT microflare occurred. Panel a): TRACE white light image with the position of the CDS slit and the DOT FoV indicated by a black and grey frame, respectively. Panel b) shows an MDI high resolution longitudinal magnetogram recorded $\sim 45$ min before the other images and rotated to 08:21:36 UT. In Panel c), a TRACE 17.1 nm image (inverse grey scale) with the same FoV as the white light map is shown. Panels d) to f) show the DOT G Band, Ca II H and H $\alpha$ images nearest in time to the TRACE white light map. In each panel, the image recording time is indicated in the top left corner.

embedded into a network cell of opposite magnetic polarity. Over the day, the spot was observed to rotate in counterclockwise direction. DOT traced the spot between $\sim 07: 44$ and 10:09 UT and acquired observations suitable for speckle reconstruction $^{2}$. The center of the CDS slit was fixed at $X=106^{\prime \prime}$ west and $Y=-149^{\prime \prime}$ south of disk center.

\subsection{Microflare detection}

During the DOT observing run we searched for microflares which occurred inside of the DOT FoV. To this end, we derived images in the 3-8 keV range of peaks observed in the RHESSI light curves and compared the position of the X-ray source to the FoV of the DOT. We found 3 RHESSI events which were also observed in the DOT H $\alpha$ and Ca II line. These three microflares also featured brightenings inside the CDS slit. The RHESSI peak times of the microflares in the 3-6 keV band are 08:26:20 UT, 08:38:10 UT and 08:45:30 UT (cf. Fig. 5). The three microflares are very small regarding their X-ray classification. Only the first of them could be determined to be of GOES class A9.2/A0.7 with/without background, whereas the other microflares were too weak to be observed by GOES. In the following, we concentrate on these three events for the detailed analysis of their

2 The processed DOT data are available in the DOT database at http://dotdb.phys.uu.nl/DOT/Data/2006_07_04/index.html chromospheric, transition region and coronal fine structure as well as their associated plasma flows.

\subsection{Coronal activity}

TRACE $17.1 \mathrm{~nm}$ maps of AR 10898 were of good quality when the first event presented here occurred $(\sim 08: 26 \mathrm{UT})$, whereas for the later two microflares at 08:38 UT and 08:45 UT, the filtergrams were spoilt by particle hits of the Earth's atmosphere.

The TRACE $17.1 \mathrm{~nm}$ data for the first microflare at 08:26 UT reveal a very complex evolution (see Fig. 2). In the course of the event, several impulsive brightenings as well as narrow loops appear. All of the TRACE brightenings probably constitute footpoints of a complex microflaring loop system (they are numbered from 1-4 in Fig. 2). The brightenings are located in the penumbra of the sunspot and in areas of enhanced magnetic flux of opposite magnetic polarity. A jet is ejected near to the northernmost TRACE brightening, close in time to the hard X-ray peak. The RHESSI 3-8 keV X-ray source associated with the event forms a small loop between the southernmost and strongest TRACE brightening situated in the penumbra $\left(X=106^{\prime \prime}, Y=-130^{\prime \prime}\right.$, footpoint no. 1) and another one to the north ( $X=110^{\prime \prime}, Y=-110^{\prime \prime}$, footpoint no. 2), thus identifying these patches as footpoints of a hot ( $210 \mathrm{MK}) \mathrm{X}$-ray 


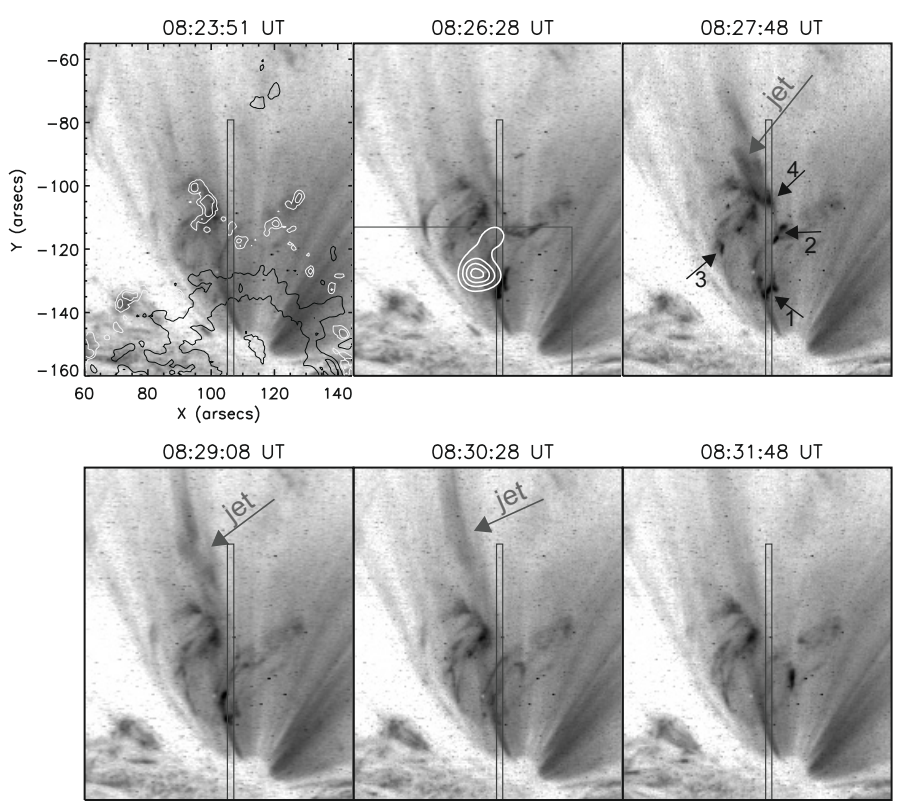

Fig. 2. TRACE $17.1 \mathrm{~nm}$ image sequence of the microflare which peaked in X-rays at $\sim 08: 26: 30$ UT. The colortable is reversed, thus bright features appear dark. In the top left panel showing the FoV before the flare started, the MDI magnetic field recorded $\sim 45$ min earlier (corrected for differential rotation) is overplotted at isocontours of $-1500,-600$, -200 (black) and $+70,+200 \mathrm{G}$ (white). The RHESSI source observed for this microflare is shown by white contours in the top middle panel (RHESSI image parameters: energy range $3-8 \mathrm{keV}$, reconstruction time 08:25:50-08:28:00 UT, contour levels at $20,50,90 \%$ of the image maximum). The position of the CDS slit is marked by a black frame in each panel. Also, we show the boundary of the DOT FoV (grey frame) for comparison with chromospheric microflare imaging in Fig. 4. We indicate the position of flare loop footpoint brightenings with black arrows and number them from 1-4. The appearance and propagation of a jet is indicated by grey arrows.

emitting loop. Another microflare brightening turns up directly on the CDS slit ( $X=106^{\prime \prime}, Y=-100^{\prime \prime}$, footpoint no. 4).

One image taken in the $19.5 \mathrm{~nm}$ passband of the EIT instrument during the late phase of the third event is available. EIT data have a spatial resolution of $\sim 5.4^{\prime \prime}$ and do not allow us to study the detailed fine structure of the event. However, a bright loop $\left(X=106^{\prime \prime}, Y=-130^{\prime \prime}\right)$ and a brightening to the north $\left(X=100^{\prime \prime}, Y=-110^{\prime \prime}\right)$ at roughly the same position as during the first event are visible. A loop-shaped RHESSI X-ray source reconstructed for energies $<8 \mathrm{keV}$ showing plasma at temperatures $T \gtrsim 10 \mathrm{MK}$ is located in between these EIT brightenings (see Fig. 3). The position of the CDS slit at the recording time of the EIT image coincides with the southern microflare brightening whereas the northern one is located at the very edge of the slit.

Although we do not have imaging information from TRACE or EIT available for the second event at 08:38 UT, we can speculate that the coronal signatures are similar to the ones of the other two microflares as all three events show homologous brightenings in the DOT H $\alpha$ and Ca II channels. For this microflare, the only imaging information about the coronal appearance comes from RHESSI which observed a $3-8 \mathrm{keV}$ X-ray source in between DOT $\mathrm{H} \alpha$ footpoints indicating the presence of a hot microflare loop (see Fig. 4).
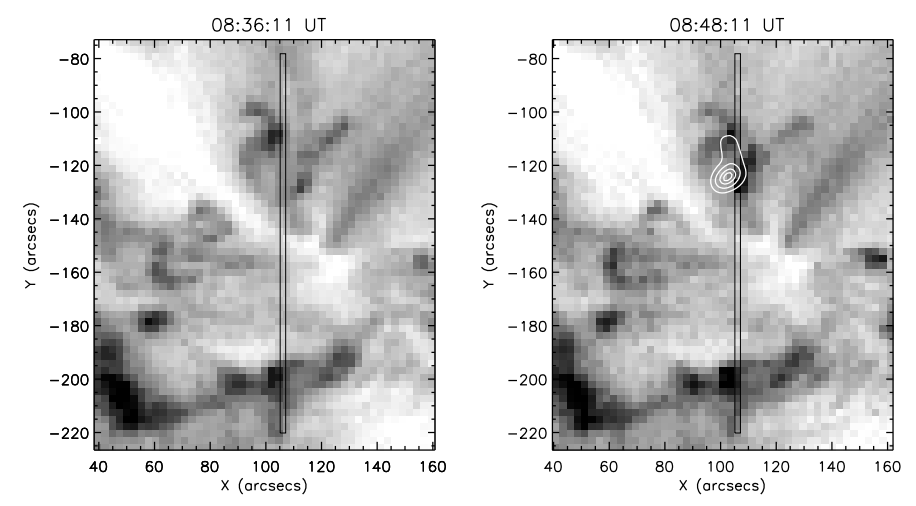

Fig. 3. EIT $19.5 \mathrm{~nm}$ maps in inverse grey scale recorded $\sim 9$ min before (left panel) and $\sim 3$ min after (right panel) the 08:45 UT microflare. The RHESSI 3-8 keV loop source observed for this event is overplotted as white contours (integration time 08:43:15-08:46:10 UT, contour levels at $30,50,90 \%$ of the maximum intensity). The position of the CDS slit is marked by a black frame. The FoV is the same as the one of the TRACE and MDI images shown in Fig. 1.

\subsection{Magnetic field environment}

For the events for which images of the whole microflare site were available (08:26 UT and 08:45 UT event), we studied the magnetic field topology in which they were formed. TRACE and DOT footpoint brightenings of the two events are found to be situated in areas of strong or enhanced magnetic flux of opposite polarities (see Fig. 2). With an MDI magnetogram time series recorded over the whole day we searched for changes in the magnetic field at the microflares' site which might facilitate reconnection like emergence/cancellation of magnetic flux (see e.g. Priest \& Forbes 2002). The large sunspot of AR 10898 was observed to rotate over the day, which will by default cause shearing of magnetic field lines which connect the sunspot with surrounding areas of opposite polarity. Magnetograms at a $1 \mathrm{~min}$ cadence taken one hour before the events show small scale $\left(\sim 2^{\prime \prime} \times 2^{\prime \prime}\right)$ magnetic flux patches moving in the sunspot penumbra and emergence/movement of flux in the opposite polarity zone where microflare loop footpoints are observed. This motion of magnetic flux could cause disturbances in the coronal magnetic field leading to the creation of microflares.

\subsection{Chromospheric response}

Whereas not the whole flaring region was covered by DOT, some of the microflare brightenings were also observed within the DOT FoV for all three events. For comparison, TRACE brightenings nos. 1-3 seen during the first event (Fig. 2) are also observed by DOT whereas brightening no. 4 is located outside the DOT FoV. During each microflare, the chromospheric DOT brightenings appeared at roughly the same position $\left( \pm 2^{\prime \prime}\right.$ difference in $x$ - and $y$-direction). The strongest brightening was always situated inside the sunspot penumbra $\left(X=106^{\prime \prime}\right.$, $\left.Y=-130^{\prime \prime}\right)$ at the southern end of the RHESSI X-ray sources. Another brightening observed for all three events was located close to the edge of the DOT FoV ${ }^{3}\left(X=110^{\prime \prime}, Y=-110^{\prime \prime}\right)$. For the first microflare, a third and weak brightening to the east $\left(X=90^{\prime \prime}, Y=-115^{\prime \prime}\right)$ was also seen. Figure 4 shows a compilation of the three microflares' time evolution in the $\mathrm{H} \alpha$ and Ca II

\footnotetext{
${ }^{3}$ In the Ca II images, a broader strip of the upper image part was cropped in the course of speckle reconstruction. Thus, this brightening could only be observed in $\mathrm{H} \alpha$ an not in Ca II.
} 

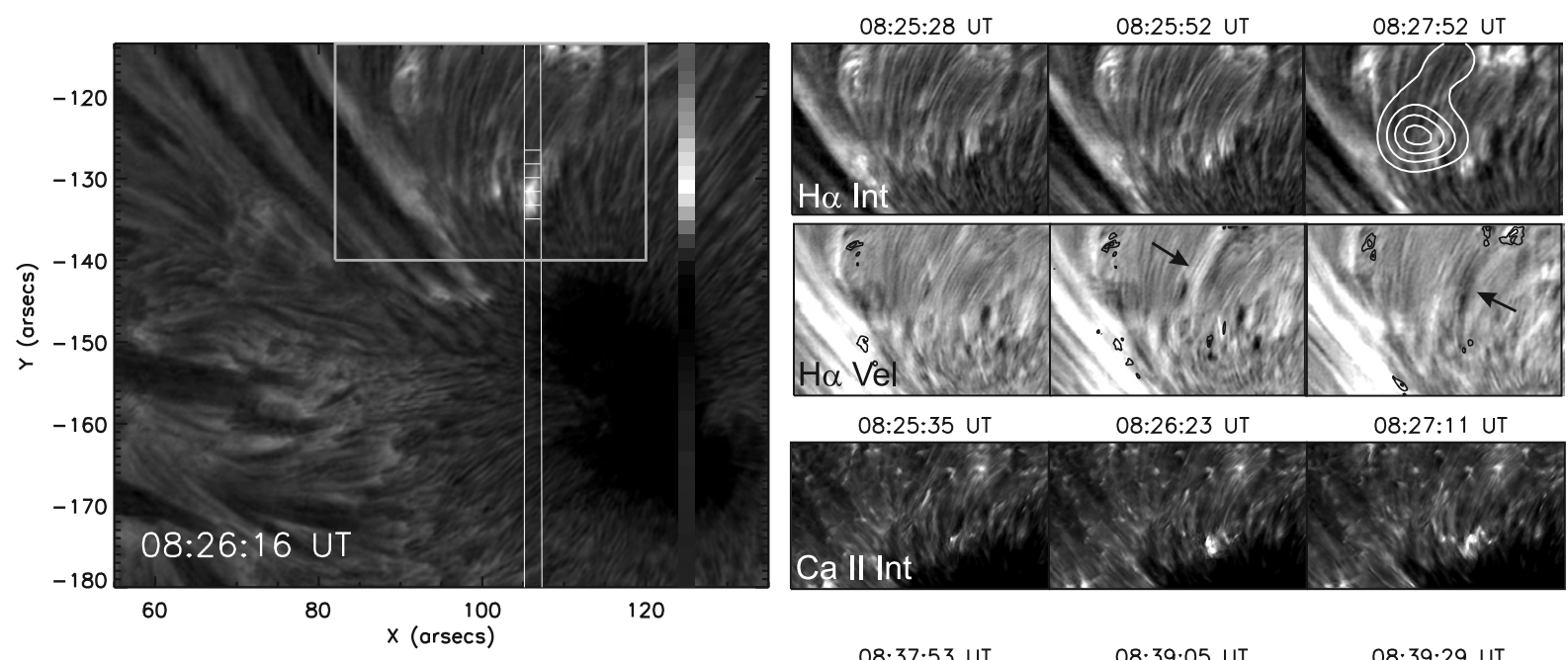

08:25:35 UT

$08: 26: 23$ UT

08:27:11 UT
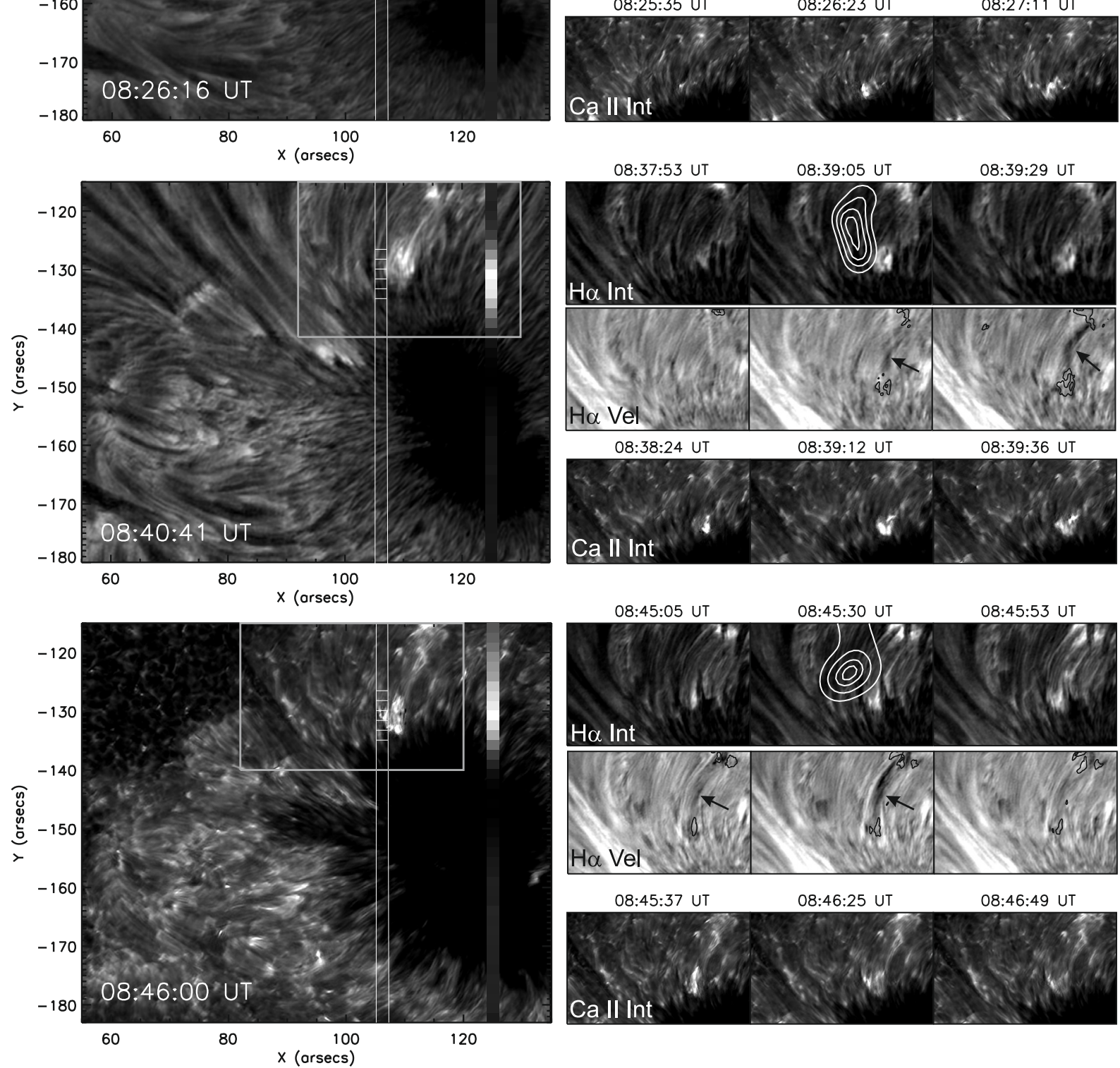

Fig. 4. Snapshots of the three microflares as observed in DOT $\mathrm{H} \alpha$ intensities and velocities as well as in the Ca II channel. On the left hand side, the whole DOT FoV in $\mathrm{H} \alpha$ (top and middle) and Ca II (bottom) is shown close to the microevents' maxima. The position of the CDS slit is indicated by white lines. An image of the slit showing the intensities in the chromospheric He I $58.4 \mathrm{~nm}$ line is overplotted with a shift of $\sim 20^{\prime \prime}$ in $x$-direction. Grey frames indicate the position and size of the subfield used to show the microflares' $\mathrm{H} \alpha$ and $\mathrm{Ca}$ II time evolution shown on the right hand side. We show the $\mathrm{H} \alpha$ intensities, Doppler velocities and $\mathrm{Ca}$ II intensities right before and for two times during the 3 microflares. We also indicate the location of the RHESSI X-ray source (3-8 keV) by white contours. For the 08:26 UT and 08:45 UT event, the image reconstruction interval and contour levels are are the same as in Figs. 2 and 3. For the second event (X-ray peak time at 08:38 UT), the RHESSI image was integrated for the time interval 08:37:55 - 08:41:10 UT and is plotted at levels of 20,50,90\% of the image maximum. The H $\alpha$ velocities are scaled to $[-5,+5] \mathrm{km} \mathrm{s}^{-1}$ (black corresponds to blueshifts and white to redshifts). In the $\mathrm{H} \alpha$ velocity images, locations of high $\mathrm{H} \alpha$ intensities are marked as black contours. The arrows in the $\mathrm{H} \alpha$ velocity maps point out fibrils showing enhanced Doppler shifts during the microflares. 
channels. The southernmost brightening in the penumbra is captured completely in all DOT channels for all events. None of the images is saturated and the full fine structure at the telescope resolution limit of $0.2^{\prime \prime}-0.3^{\prime \prime}$ is exhibited. The brightenings show fine sub-structure with patches of enhanced emission sized down to $1^{\prime \prime} \times 1^{\prime \prime}$ (see Fig. 4). Interestingly, for some times during the events, the $\mathrm{H} \alpha$ brightenings are partly hidden behind dark fibrils which show enhanced Doppler shifts (cf. Fig. 4, H $\alpha$ intensities and velocities at 08:45:30 UT). Most noticeably in the third event, fibrils brighten up in between the footpoint brightenings (Fig. 4, image at 08:45:53 UT). The connectivity of the magnetic field at the microflare site can be estimated by the shape of chromospheric fibrils which closely follow the shape of the RHESSI X-ray sources observed for the first and second event. Approximately $3 \mathrm{~min}$ after the southern footpoint of the third event has faded, dark "threads" from outside the DOT FoV are observed to flow down to the position of the former microflare brightening in the red wing of the $\mathrm{H} \alpha$ line, maybe due to the restructuring of the magnetic field on a larger scale, although the formation and origin of these flows is unclear.

Compared to the dynamic range of the $\mathrm{H} \alpha$ emission, the flare brightenings observed in the $\mathrm{Ca}$ II line are much more intense than the quiet sun emission and fade away faster. The brightenings have a spikier appearance than in $\mathrm{H} \alpha$. Most visible in the 08:45 UT event but also observed for the other two, a bright, loop-shaped feature in between the southern penumbral footpoint and the brightening at the edge of the DOT FoV appears. This feature looks similar to the bright fibrils observed in the $\mathrm{H} \alpha$ line.

All the $\mathrm{H} \alpha$ and $\mathrm{Ca}$ II microflare brightenings are found to be dynamic. They were moving for a small amount (on the order of $<5^{\prime \prime}$ ) during their life time. According to the eruptive standard flare model, chromospheric flare loop footpoints appear moving with time further away from the magnetic neutral line. Although the DOT brightenings observed during the microevents are moving, they do not clearly separate from each other as is generally observed for large two ribbon flares. However, they are of very short duration and occur in a complex small-scale magnetic field topology which could be the reason why no clear separation is observed.

A movie combining DOT images from the G band, $\mathrm{H} \alpha$ (intensities and velocities), Ca II intensities as well as RHESSI data of the microflares is available as online supplementary material (see Appendix A for a description).

\section{Associated plasma flows}

\subsection{CDS - flows at chromospheric, transition region and coronal temperatures}

For each of the 3 microflares, the CDS instrument observed 2 brightenings in all lines, situated at roughly the same location on the slit. They are located in the range $-110^{\prime \prime} \lesssim$ $Y_{1} \lesssim-95^{\prime \prime}$ and $-140^{\prime \prime} \lesssim Y_{2} \lesssim-125^{\prime \prime}$. In the following, we will refer to them as to the "northern" brightening or "southern"/"penumbral" brightening (as it is situated in the penumbra). See Fig. 2 for a comparison how they appear in spatially resolved TRACE data recorded for the first microflare (footpoints nos. 1 and 4). Although the DOT and TRACE brightenings corresponding to the CDS brightenings are very small $\left(\sim 2-5^{\prime \prime}\right)$, the CDS brightenings cover several CDS pixels (5-20", see Figs. 5 and 6). The microflare brightenings were not always located at the position of the CDS slit in the impulsive phase (i.e. at the time of largest energy input) which is important to keep in mind

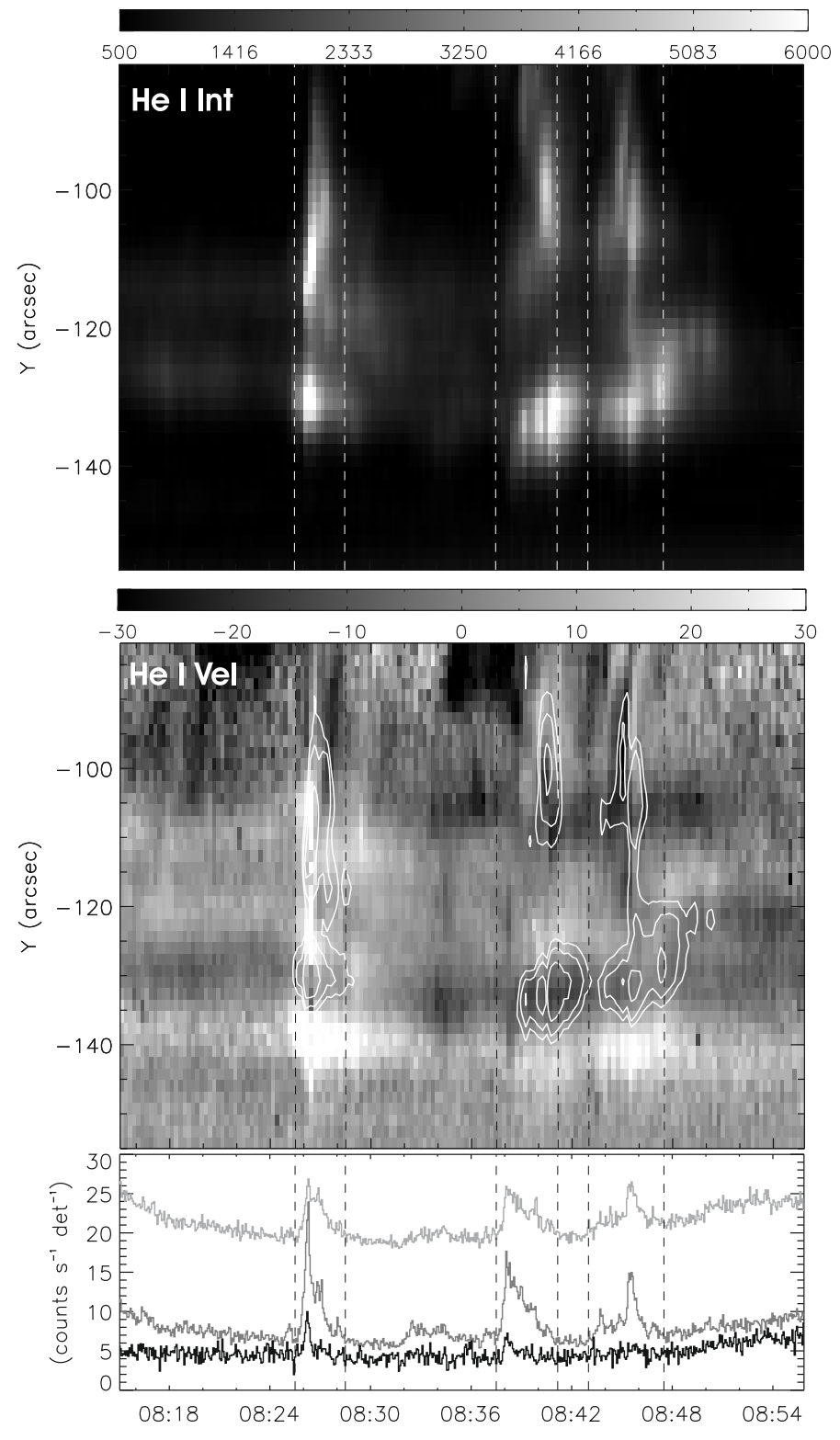

Fig. 5. Top: specific intensities along the slit in the He I line, i.e. the amplitudes of the broadened Gaussian fit applied to the line profiles in each pixel (units of erg $\mathrm{cm}^{-2} \mathrm{~s}^{-1}$ sterad $^{-1} \AA^{-1}$ ). Middle: same as the top panel but the velocities are plotted in the range $[-30,+30] \mathrm{km} \mathrm{s}^{-1}$ (black indicates blue- and white redshifts). For better comparison with the top panel, contours mark the positions of high He I intensity. The intensities and velocities are derived from one component broadened Gaussian fits to the observed line profiles. Bottom: RHESSI light curves in the energy bins 3-6 keV (light grey), 6-9 keV (dark grey) and 9-12 keV (black). In each panel, black or white dashed lines mark the start and end of RHESSI X-ray emission in the microflares.

when interpreting the results. For the first event, the northern and southern brightening lie directly inside the CDS slit (see Figs. 2 and 4). For the second event, the timing of the RHESSI light curve and the evolution of the CDS intensities in all lines suggests that CDS observed the microflare only in the X-ray decline phase (cf. Fig. 5). For the third microflare, both the southern and northern brightening are observed at the flare peak. However, it seems that only the edges of both brightenings are located in the CDS slit (Fig. 3 and 4). As CDS data show an abundance of information not only confined to bright microflare pixels, we first illustrate the velocity field along the whole CDS slit for $\sim 45 \mathrm{~min}$ 


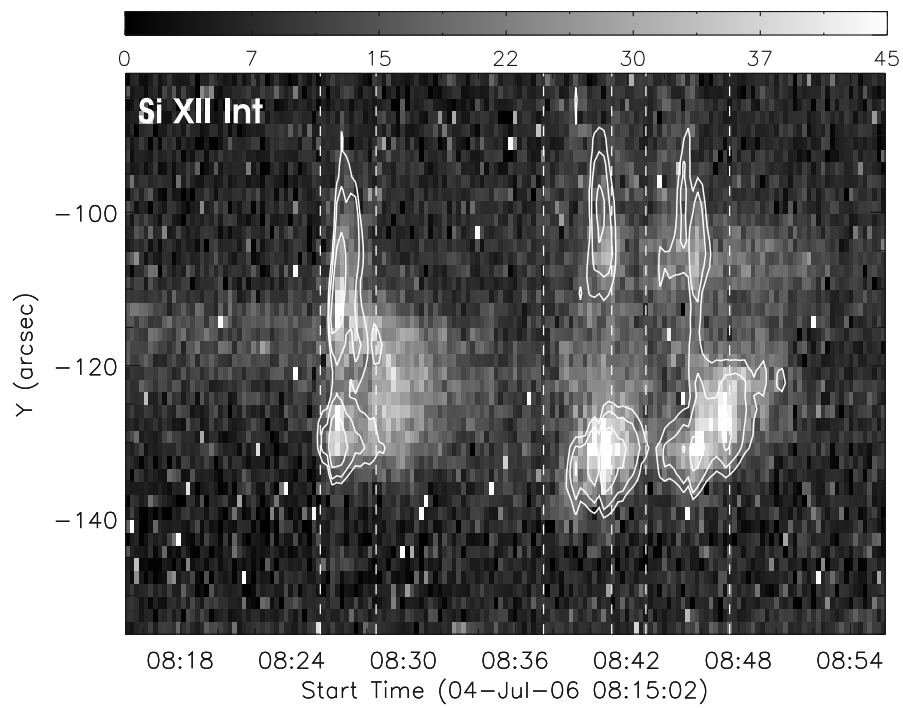

Fig. 6. The evolution of intensities (units of erg cm $\mathrm{cm}^{-2} \mathrm{~s}^{-1} \operatorname{sterad}^{-1} \AA^{-1}$ ) in the $\mathrm{Si}$ XII line $(T \approx 2 \mathrm{MK})$ are plotted for the same time interval as in Fig. 5. White dashed lines mark the beginnings and ends of the three microflares in RHESSI X-rays. White contours indicate high He I intensities.

with a CDS space-time plot. Afterwards, details on the intensity and velocity development in selected pixels inside the microflare brightenings are shown. In the following text and all figures showing CDS observations, "intensities" mean the amplitude of the broadened Gaussian function fitted to the line profiles (units of erg cm $\mathrm{cm}^{-2} \mathrm{~s}^{-1} \mathrm{sr}^{-1} \AA^{-1}$, i.e. the specific intensity).

Figure 5 shows the RHESSI X-ray emission in the range 3-12 keV together with the time evolution of the intensities and velocities along the CDS slit in the chromospheric He I $58.43 \mathrm{~nm}$ line. This CDS space-time plot has the advantage that flows associated with the events occurring in pixels in- as well as outside the main flare brightenings become visible. The general flow pattern observed in this chromospheric line is complicated. For the 08:26 UT microflare, the start and end of enhanced emission closely follows the RHESSI light curve. He I downflows are observed at the position of the northern and southern flare brightenings as well as in between them. However, the strongest downflows within the penumbral brightening are observed two or three pixels to the north from the intensity maximum, whereas only small velocity enhancements are found in the brightest pixels. In the northern brightening, approximately at the time of a second small peak in the RHESSI light curves, blueshifts indicating upflow velocities of $v \approx-20 \mathrm{~km} \mathrm{~s}^{-1}$ appear at $Y \approx-105^{\prime \prime}$. These upflows "move" with time further to the north, forming a streak in the CDS space-time plot. This might be a signature of the jet seen by TRACE to move out of the corona close to the northern footpoint brightening (Fig. 2). During the second event, the He I line is blueshifted in the entire southern brightening indicative of upflows whereas in the northern brightening, blueshifts can only be observed in pixels with low intensities at the lower edge. For the third event, first blueshifts and a fast change to redshifts can be seen at the position of the northern footpoint whereas weak downflows are observed for the southern brightening. The reason why we do not a priori see the strongest flows in the brightest pixels might be a geometry effect as we can only observe the line of sight velocity. On the other hand, this could also have a physical reason, e.g. the strongest flows occur at the edge of the microflare brightenings.
Flows associated with the microflare events, however, are not only confined to pixels with high emission. Strong downflows which reach speeds of $\sim+70 \mathrm{~km} \mathrm{~s}^{-1}$ are observed up to $\sim 10^{\prime \prime}$ to the south of the penumbral footpoint. During all three events, such enhanced downflows into the sunspot penumbra occur. Similar downflows are also observed in the $\mathrm{O} \mathrm{v}$ line.

In the coronal Si XII line ( $T \sim 2 \mathrm{MK})$, high intensities are found at the location of the two chromospheric He I brightenings which mark heated flare loop footpoints (Fig. 6). With time, pixels in between these brightenings become more intense, probably showing emission from a hot flare loop located in between the chromospheric microflare brightenings. However, no blueshifts which would indicate the motion of matter from the chromosphere into the corona are observed for the Si XII line which is at rest during all three events. This could be due to low count statistics in Si XII. On the other hand, we could observe heating of the footpoints of the flare loops early in the events followed by cooling of hotter plasma created during the flare into the $2 \mathrm{MK}$ range. The presence of plasma hotter than $2 \mathrm{MK}$ is proved by RHESSI X-ray spectra derived at the microflares' peak which can be fitted at the lowest energies by an isothermal bremstrahlung component with a temperature around 10 MK. Studying four regular flares, Kamio et al. (2005) found the Mg IX line $(T \sim 1 \mathrm{MK})$ formed at similar temperatures as the Si XII line also to be at rest. They argued that this might indicate that the line is formed at an intermediate temperature where neither up- nor downflows can be observed. This could also apply to the Si XII line in these microflares.

Details on the velocity signal in chromospheric and transition region microflare brightenings are shown in CDS light curves derived for individual bright pixels. In Figs. 7 to 9, we show for each event the time evolution of CDS He I and O V intensities and velocities in selected pixels of the northern and southern brightening. We concentrate on the He I and $\mathrm{O} \mathrm{V}$ line as they have the best signal to noise ratio, and the line fits are also reliable for quiet sun areas. Velocity enhancements observed in other lines are only discussed in the text. For the first microflare, the $\mathrm{He} \mathrm{I}, \mathrm{O} \mathrm{V}$ and $\mathrm{Ne}$ VI lines are redshifted for both northern and southern brightening indicating plasma flows of $\sim+10$ $40 \mathrm{~km} \mathrm{~s}^{-1}$. For the second event at 08:38 UT, chromospheric upflows both in $\mathrm{He} \mathrm{I}\left(-10 \mathrm{~km} \mathrm{~s}^{-1}\right), \mathrm{O} \mathrm{V}\left(-40 \mathrm{~km} \mathrm{~s}^{-1}\right)$ as well as in the hotter $\mathrm{Ne}$ VI line $\left(-40 \mathrm{~km} \mathrm{~s}^{-1}\right)$ are observed in the penumbral brightening (cf. Fig. 8). In the northern brightening, no line shows a clear velocity signal in the brightest pixels and upflows are only observed at the lower edge of the brightening (He I: $\lesssim-20 \mathrm{~km} \mathrm{~s}^{-1}, \mathrm{O} \mathrm{v}: \lesssim-50 \mathrm{~km} \mathrm{~s}^{-1}$ ). Event no. 3 which peaked in X-rays at $\sim 08: 45: 30$ UT shows the smallest RHESSI peak flux. The flow speeds and directions suggested by CDS line fits indicate upflows reaching velocities of at most $-30 \mathrm{~km} \mathrm{~s}^{-1}$ in the northern brightening ( $\mathrm{He} \mathrm{I}, \mathrm{O}$ V and $\mathrm{Ne} \mathrm{VI}$ ) and weak downflows of up to $+20 \mathrm{~km} \mathrm{~s}^{-1}$ in the southern footpoint (He I and O v, see Fig. 9).

Line profiles were for most of the time well fitted with a one component broadened Gaussian. However, for a short time right at the microflares' maxima (1-3 CDS exposures), the CDS profiles of several lines show two or even three peaks. For the 08:26 UT event, such multi-component profiles are observed in He I, O V and Ne VI at as well as in between the northern and southern brightening. Double component fits to these spectra indicate strong downflows of $\sim+180 \mathrm{~km} \mathrm{~s}^{-1}$ and upflows up to $\sim-80 \mathrm{~km} \mathrm{~s}^{-1}$ within the same pixel area (see Fig. 10). As these double component profiles are not only observed at the footpoint brightenings but also in between the northern and southern CDS brightening, these oppositely directed flows could 

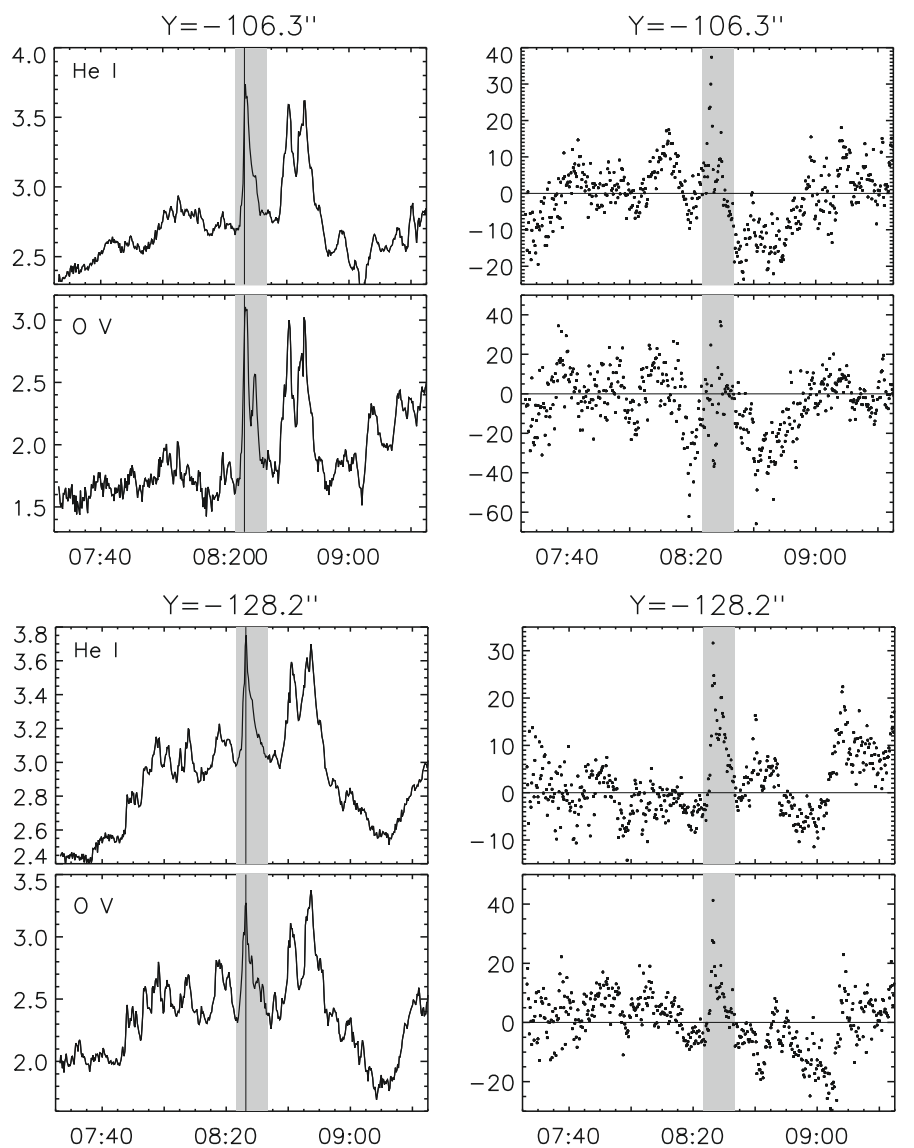

Fig. 7. For the first event, the time development of intensities and velocities in the CDS He I $\left(T \approx 3.9 \times 10^{4} \mathrm{~K}\right)$ and $\mathrm{O}$ V line $\left(T \sim 2.6 \times 10^{5} \mathrm{~K}\right)$ observed in selected pixels at the northern (top) and southern brightenings (bottom) is plotted. The grey highlighting marks the life time of the events as seen by CDS. The $Y$-position along the slit of the pixel for which the light curves and velocity evolution were derived is indicated in the title (units of arcsec from disk center). The intensities (solid lines) have units of erg $\mathrm{cm}^{-2} \mathrm{~s}^{-1}$ sterad $^{-1} \AA^{-1}$ and are plotted on logarithmic scale. Velocities (crosses) are given in units of $\mathrm{km} \mathrm{s}^{-1}$ where positive numbers denote redshifts and negative values blueshifts of the line. The time of maximum in RHESSI X-rays is indicated in the intensity curves by a vertical line.

indicate a twisting motion of the flare loop. Multi component profiles suggesting twist motions are also observed in the second event where they occur in several bright pixels and in several lines (He I, O III and $\mathrm{O}$ V at the northern footpoint, upand downflow velocities $\lesssim 160 \mathrm{~km} \mathrm{~s}^{-1}$ ). Also for the third event, two-component profiles of the $\mathrm{O} \mathrm{V}$ line right at the penumbral as well as the northern footpoint indicate up- and downflows of $\sim-70$ and $+100 \mathrm{~km} \mathrm{~s}^{-1}$ within the same pixel area.

\subsection{DOT - chromospheric flows}

The DOT Doppler maps derived from $\mathrm{H} \alpha$ wing images also show flows associated with the microflares. Most prominent, fine loop-shaped fibrils in between the footpoints are Doppler shifted during the events. Up- as well as downflowing plasma with speeds of the order of $5 \mathrm{~km} \mathrm{~s}^{-1}$ appear in very close proximity to each other (see Fig. 4). At the positions of $\mathrm{H} \alpha$ footpoints, however, rarely any change in flow speed is observed. Only for the first microflare at 08:26:30 UT, H $\alpha$ downflows appear at the footpoint close to the edge of the DOT field of view (Fig. 4, $\mathrm{H} \alpha$ velocity map at 08:27:52 UT). This is interesting as
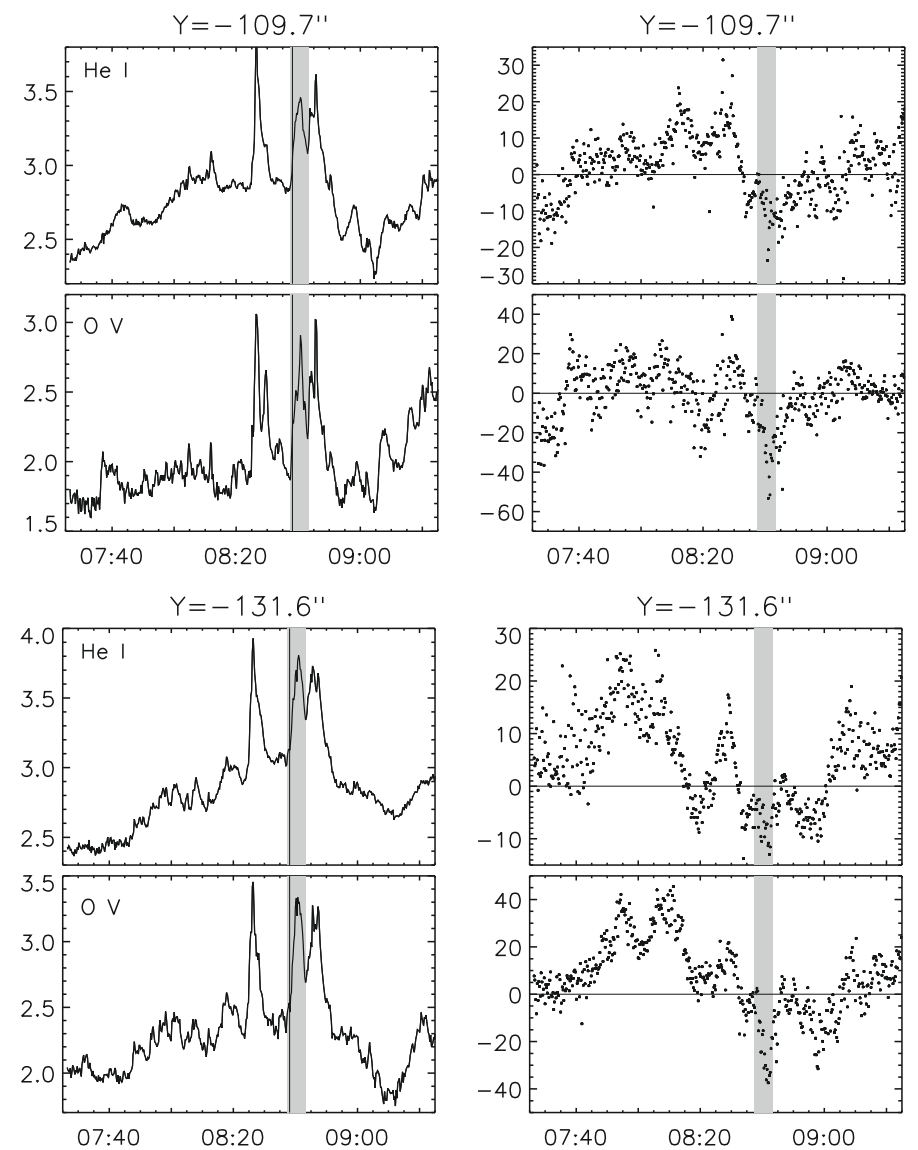

Fig. 8. Same as Fig. 7 for the microflare at 08:38 UT.
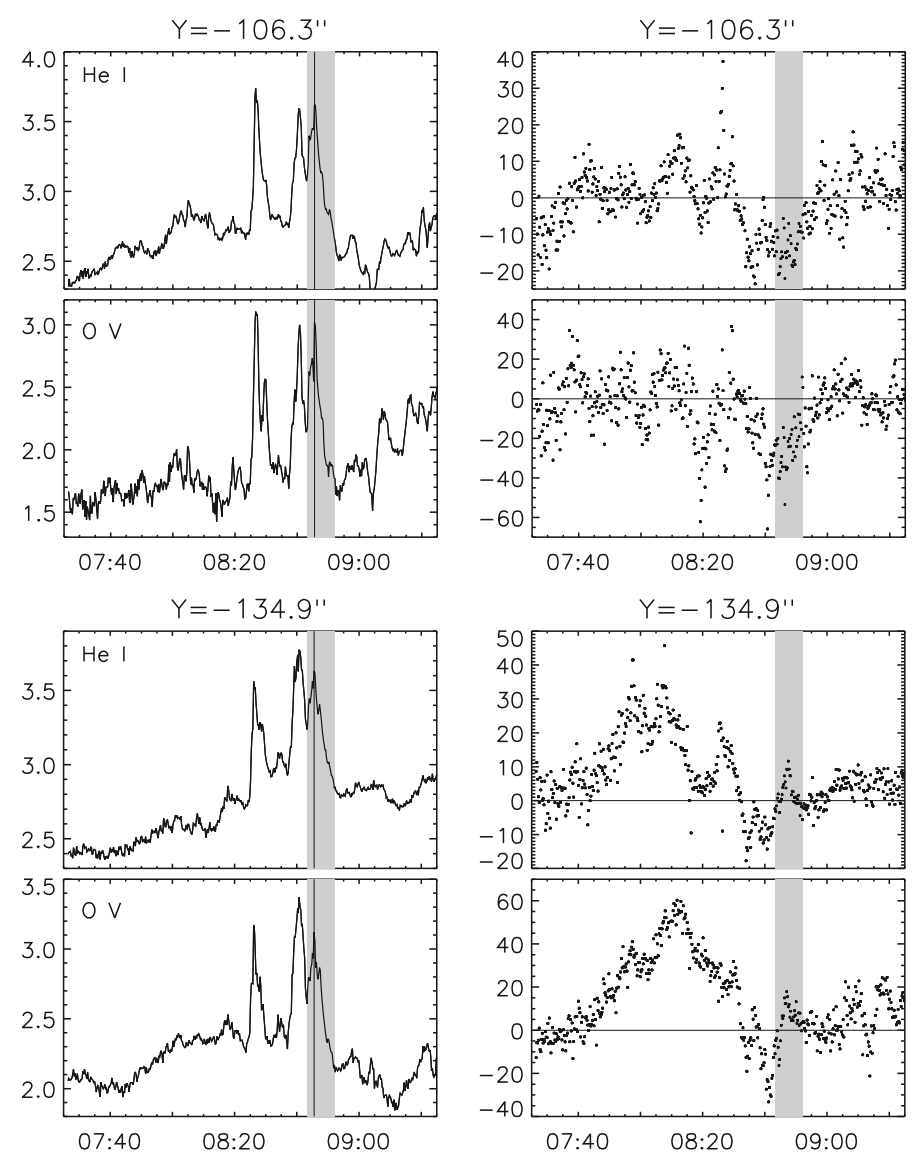

Fig. 9. Same as Fig. 7 for the microflare at 08:45 UT. 

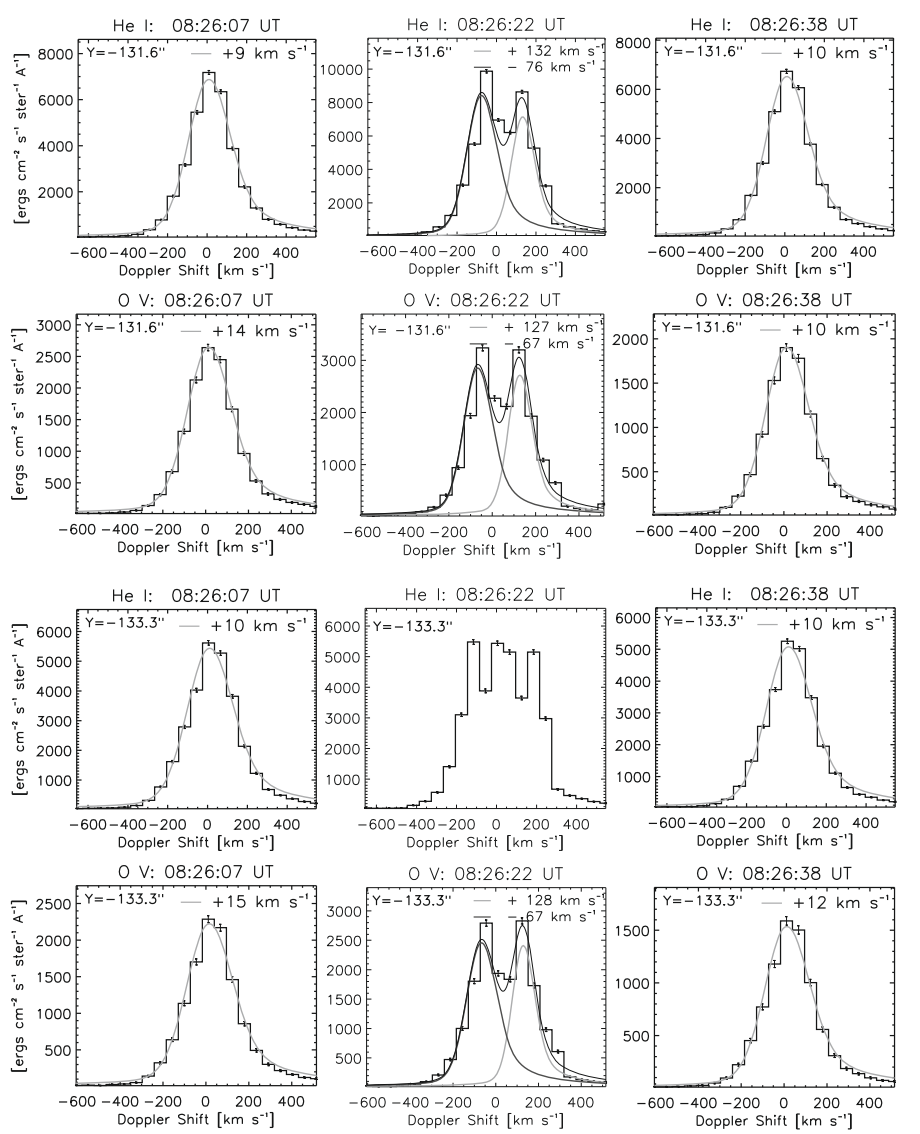

Fig. 10. He I and $\mathrm{O} v$ line spectra recorded in the penumbral footpoint of the 08:26 UT event at the time of highest intensity (middle column) as well as in the preceding and following exposure. We show the profiles for 2 different pixels $\left(Y=-131.6^{\prime \prime}, Y=-133.3^{\prime \prime}\right)$. The grey solid lines mark broadened Gaussians which were fitted to the profiles in order to derive Doppler velocities. Where applicable, a second component was fitted (black, bold line) and the sum of both Gaussians is plotted as a thin black line. The results for the fitted line of sight velocities are given in the top right corner of each panel. Note, that the He I line at 08:26:22 UT shows even three components in the $Y=-133.3^{\prime \prime}$ pixel.

the chromospheric He I line profiles recorded at the position of flare brightenings are Doppler shifted for each flare. The reason for the "missing" flows at high intensity areas in $\mathrm{H} \alpha$ may be that the line profiles at the footpoints have a complex shape due to heating and thus, reliable Doppler shifts cannot be derived from only two sample points (wings) of the spectral line.

\section{RHESSI X-ray spectroscopy}

Based on RHESSI spectra, information on the temperature and emission measure of the hottest part of the microflare plasma can be gained. Also, we searched for signs of non-thermal bremsstrahlung emission indicating the presence of electron beams. For each event, we derived the spectra at a binning of $1 / 3 \mathrm{keV}$ integrated over $12 \mathrm{~s}$ at the peak of the RHESSI X-ray light curves (see Fig. 11). Energy bins of $1 / 3 \mathrm{keV}$ are actually only correct for spectroscopy with single detectors whereas for our microflares we derived spectra summed over all detectors excluding detectors 2 and 7. However, when analyzing spectra for the same time intervals which have the nominal spectral resolution of $1 \mathrm{keV}$, we got very similar results (as was also found by Qiu et al. 2004). Therefore, we here show the RHESSI spectra binned at $1 / 3 \mathrm{keV}$. In RHESSI spectral analysis software, a user defined model spectrum is fitted to the observed data where the selected model is not a unique choice. The quality of the fit (expressed in terms of the $\chi^{2}$ quantity) can be used to distinguish which models are physically appropriate. For the first and second event, the spectra were fitted with a two component model consisting of an isothermal bremsstrahlung component at the lowest energies and a non-thermal power-law including a low-energy cutoff at $\sim 9 \mathrm{keV}$. A purely thermal fit for these events was of very poor quality with $\chi^{2} \gtrsim 4$ whereas for the 2 -component fit $\chi^{2} \lesssim 1$. Thus, the combination of a thermal and non-thermal bremsstrahlung model spectrum is certainly to be preferred to a solely thermal fit. In contrast, the peak spectrum of the third event was acceptably fitted with a thermal fit alone $\left(\chi^{2} \approx 2\right)$ and showed only weakly enhanced flux at energies $\gtrsim 9 \mathrm{keV}$ relative to the purely thermal fit. The derived emission measures $\left(E M \approx 10^{45} \mathrm{~cm}^{-3}\right)$ and temperatures $(T \approx 10-13 \mathrm{MK})$ of the flare plasma lie in the range generally found for RHESSI microflares (Hannah et al. 2008). However, the EM lie at the low end of the observed distribution, indicating that we deal with very small events.

Clear signatures of a non-thermal power-law spectrum in the first and second event indicate the presence of beamed electrons accelerated during the reconnection process. This suggests the possibility that the observed plasma flows are caused by beam driven chromospheric evaporation. The non-thermal bremsstrahlung fit to the power-law spectra yielded photon spectral indices of $\gamma \approx 4.2$ and $\gamma \approx 5.2$ in the 08:26 UT and 08:38 UT event, respectively. These are rather hard power-law spectra for such weak events. Analyzing the spectra of thousands of RHESSI microflares, Hannah et al. (2008) reported a median value of $\gamma \approx 7$. The non-thermal component is of special interest as it allows us to estimate the energy of supra-thermal electrons deposited in the chromosphere which determines the characteristics of chromospheric evaporation, i.e. whether it is "explosive" or "gentle". Although the non-thermal electron power in the microflares is by orders of magnitude smaller than in regular flares, we observe very small footpoint areas. This means that the non-thermal energy flux density can nevertheless be high. For the events for which a non-thermal spectrum is observed, we can determine if flux densities on the same order of magnitude as serve as input for flare simulations can also be attained in microflares. If so, a substantially lower amount of non-thermal flux density in the second than in the first microflare would be support coming from the RHESSI analysis that the threshold between gentle and explosive evaporation is surpassed in the first event. In the simulations of Fisher et al. (1985b), the critical heating flux separating the gentle and explosive evaporation regime is estimated to $\sim 10^{10} \mathrm{erg} \mathrm{cm}^{-2} \mathrm{~s}^{-1}$. With the fits of the RHESSI power-law spectra in the first and second event, we can determine the non-thermal power of the beam at the flare peak. The low energy cutoff of the beams was found to be situated at $\sim 8-10 \mathrm{keV}$. We assume that all electrons with energies $\gtrsim 10 \mathrm{keV}$ reach the chromosphere (see also Stoiser et al. 2008). This is certainly a rather generous estimate and constitutes an upper boundary. The power of the beam above $10 \mathrm{keV}$ for the two events is $P_{10,1} \approx 1.4 \times 10^{26} \mathrm{erg} \mathrm{s}^{-1}$ and $P_{10,2} \approx 1.1 \times 10^{26} \mathrm{erg} \mathrm{s}^{-1}$. To derive the electron flux density, we need the impact area of the beams which we estimated from DOT or TRACE images at the flare peak. For simplicity, we assumed that the beam in both microflares is incident on an area twice the size of the penumbral footpoint observed in 

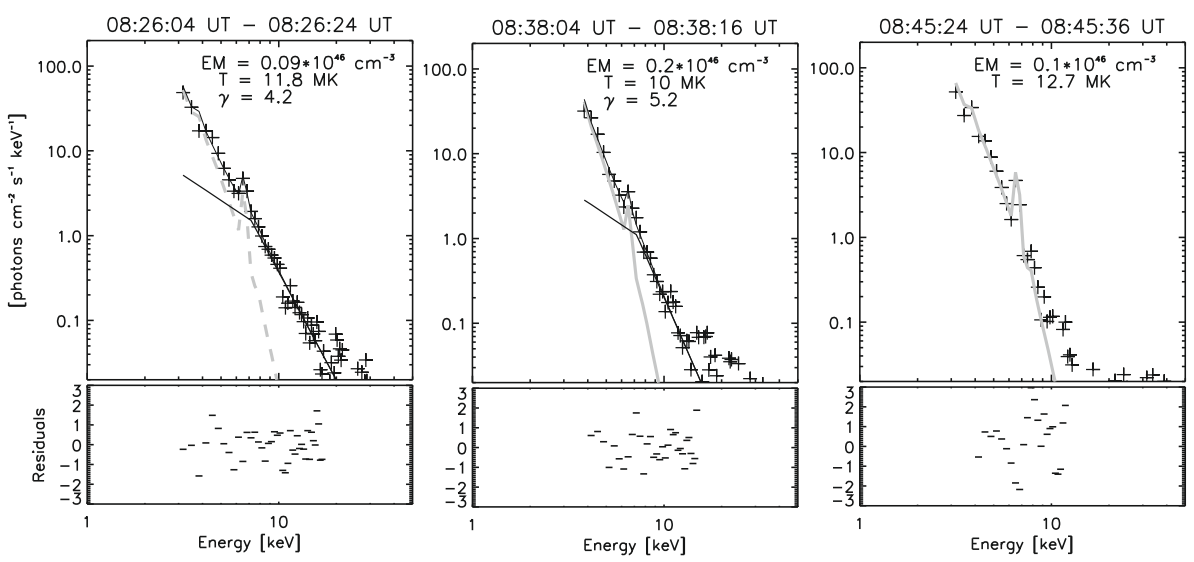

Fig. 11. RHESSI photon spectra at an energy resolution of $1 / 3 \mathrm{keV}$ (crosses) derived at the $\mathrm{X}$-ray peak of the three analyzed events. The spectra for the 08:26 UT and the 08:38 UT event were fitted with two components: a thermal bremsstrahlung model at low energies (dashed grey line) and a non-thermal powerlaw at energies $\gtrsim 9 \mathrm{keV}$ (bold solid line). The sum of both components is plotted as a thin solid line. For the third event at 08:45 UT, the spectrum was fitted by a thermal model alone. The normalized residuals are given for each spectrum shown. The fitted emission measure EM and temperature $T$ as well as the photon spectral index $\gamma$, where applicable, are indicated for each event. The bump in the spectra between 6 and $8 \mathrm{keV}$ is due to an (unresolved) iron line complex centered around $6.7 \mathrm{keV}$. the DOT $\mathrm{H} \alpha$ filtergrams. For the first event, this area ${ }^{4}$ is estimated to $5 \times 10^{15} \lesssim A_{\mathrm{f}} \lesssim 4 \times 10^{16} \mathrm{~cm}^{2}$. With these limits for $A_{\mathrm{f}}$, the beam flux density for the 08:26 UT event ranges in between $0.4 \times 10^{10} \lesssim F_{1} \lesssim 2.8 \times 10^{10} \mathrm{erg} \mathrm{cm}^{-2} \mathrm{~s}^{-1}$. For the 08:38 UT event, we derived limits to the footpoint area of $4 \times 10^{15} \lesssim A_{\mathrm{f}} \lesssim 2 \times 10^{16} \mathrm{~cm}^{2}$. The non-thermal flux density $F_{2,10}=P_{10,2} / A_{\mathrm{f}}$ is thus $0.6 \times 10^{10} \lesssim F_{2} \lesssim 2.8 \times 10^{10} \mathrm{erg} \mathrm{cm}^{-2} \mathrm{~s}^{-1}$. The numbers for the non-thermal electron energy flux density are very similar for both events and, within their respective uncertainties, straddle the theoretical threshold $\left(\sim 10^{10} \mathrm{erg} \mathrm{cm}^{2} \mathrm{~s}^{-1}\right)$ between explosive and gentle evaporation (Fisher et al. 1985b). So we cannot conclude from X-ray observations that there exists a clear distinction between the first and second microflare which would explain their different flow behavior in the chromosphere and transition region. However, we have shown that energy flux densities sufficiently high to produce explosive evaporation may occur in very small flares, consistent with the observation of downflows in the impulsive phase of microevents.

For the third event, we have no clear spectral signs of nonthermal electrons. Certainly, the absence of a non-thermal spectrum could be also due to insufficient sensitivity of the RHESSI detectors for such a small event. So beam driven evaporation is not ruled out as a process to cause the observed flows. However, beam evaporation is not the only process in which hot RHESSI flare plasma can be produced. Direct heating of coronal preflare plasma to RHESSI temperatures and subsequent conductive chromospheric evaporation is also a process to be considered. In this scenario, the origin of chromospheric and transition region downflows in the southern flare brightening in $\mathrm{He} \mathrm{I}$ and $\mathrm{O} \mathrm{V}$ is, however, unclear.

\section{Discussion}

Observations of three microflares in the chromosphere, transition region and corona at high spatial and temporal resolution reveal a wealth of information on the dynamic evolution of microflares. It is, however, a challenge to combine all particulars into a coherent, general picture of the processes going on in the events with respect to the standard flare model.

We analyzed three homologous microevents of GOES class $<$ A9 which peaked within several minutes from each other. The general multi-wavelength properties and magnetic field topology of the three events are in basic agreement with the ones

${ }_{4}$ The lower and upper limits of $A_{\mathrm{f}}$ were derived from DOT H $\alpha$ difference images and the plain $\mathrm{H} \alpha$ maps at the flare peak, respectively. observed for regular flares and microflares, believed to be created by magnetic reconnection. Imaging information (TRACE and EIT maps) with high resolution of the entire flaring region are only available for the first and third event. TRACE $17.1 \mathrm{~nm}$, maps of the first event at $\sim 08: 26$ UT reveal a complex flare sequence with several footpoint brightenings and narrow loops. An EUV jet is ejected at the event peak of the first microflare, revealing the eruptive nature of the event. The chromospheric fine structure is exhibited in DOT Ca II and $\mathrm{H} \alpha$ images which outline brightenings with substructure to $\sim 1^{\prime \prime} \times 1^{\prime \prime}$. In both chromospheric lines, loop-shaped fibrils lying in between the footpoints are observed to brighten up at the flare peak. For all three events, RHESSI observed a hot loop $(\sim 10 \mathrm{MK})$ in between the footpoint brightenings.

Using spectroscopic data from CDS sampling the chromosphere, transition and corona, we searched for mass flows in the solar atmosphere associated with the microflares. However, although the multi-wavelength properties are similar for the three microflares, their flow dynamics is different. As expected from chromospheric evaporation theory, enhanced plasma flows could indeed be observed. CDS EUV line spectra reveal a very complex flow pattern at the site of CDS He I, O V and Ne VI microflare brightenings themselves as well as for adjacent, dark pixels. Except for few seconds at the flare peak where we observe double component line profiles, the flow speeds in the brightest pixels in chromospheric and transition region lines do not surpass $\sim 50 \mathrm{~km} \mathrm{~s}^{-1}$. The direction of the flows differs among the events themselves as well as for the footpoints within the same microflare in the third event: downflows are observed for both penumbral and northern footpoint of the 08:26 UT event as well as in the penumbral footpoint of the event at 08:45 UT. On the other hand, upflows are most prominent at the penumbral footpoint of the 08:38 UT event and are also observed at the northern footpoint of the third event. When judging from the chromospheric and transition region flows, the observations in the first event are in agreement with the explosive evaporation scenario whereas in the second event, gentle evaporation is implied. On the other hand, the flows in the third microflare are more complex and even suggest a different behavior for different footpoints.

The coronal response, however, is not at first sight in line with the results for the chromosphere and transition region. The hottest available CDS line formed at coronal temperatures (Si XII, $T \sim 2 \mathrm{MK}$ ) is not Doppler shifted during any microflare. Thus, we lack clear evidence that hot plasma is transported from the chromosphere to the corona. Maybe, the "missing" upflows 
indicate that hot, coronal flare plasma in these events is rather produced by direct heating than chromospheric evaporation (see e.g. observations of Falchi et al. 2006 and Milligan 2008). On the other hand, the Si XII line could be formed at a temperature too cool to observe upflows as argued by Kamio et al. (2005). Also, the signal to noise ratio of this weak line is maybe too low in our microflares to get a clear velocity signal.

As RHESSI X-ray spectra for the first and second microflare show a non-thermal power-law component indicating the existence of beamed electrons, we were able to estimate the nonthermal electron flux density deposited in the chromosphere. Simulations of beam driven chromospheric evaporation computed by Fisher et al. suggest explosive evaporation above a certain threshold heating flux which should be observable by redshifts of chromospheric and transition region lines. The electron energy flux density incident on the chromosphere in two microflares was found to be high $\left(F \approx 10^{10} \mathrm{erg} \mathrm{cm}^{-2} \mathrm{~s}^{-1}\right)$ which is right at the threshold between the gentle and the explosive evaporation regime. When considering the uncertainties contained in the observations (errors in X-ray spectra, impact areas) as well as in simulations (e.g. model assumptions), we cannot clearly discriminate if the electrons induce gentle or explosive evaporation. As the third event did not show a clear non-thermal component in the spectrum, we do not have distinct support that the observed mass flows can be caused by the impact of electron beams. Certainly, limited instrument sensitivity could account for missing indications of non-thermal emission. Also, as argued by Brosius \& Holman (2009), the simultaneous peak of the RHESSI X-ray and chromospheric He I emission is consistent with energy deposition of non-thermal electrons. However, the process causing chromospheric flare brightenings and mass flows in the 08:45 UT event could also be direct heating of coronal plasma and subsequent conductive evaporation.

Apart from the general flow behavior at the flare brightenings, we found many details in the time evolution of the spectra as well as flows at locations not immediately connected with high intensities. An intriguing behavior of the CDS EUV lines is observed during a short period (typically only for one exposure) right at the peaks of the microflares. In all events, line profiles (He I, O v for all 3 events, Ne VI for the first and $\mathrm{O}$ III for the last event) located at the flare brightenings themselves as well as in between them show two or three peaks indicating oppositely directed flows within the same pixel area. Fits yield red- and blueshifts of up to $\sim+190$ and $\sim-80 \mathrm{~km} \mathrm{~s}^{-1}$, respectively. The appearance of multi-component profiles is coupled with times of high energy deposit in the microflares. As multi-component profiles are observed in several pixels between northern and penumbral brightenings, we possibly see twisting motions of the flare loop. Maps of the Doppler velocities in the $\mathrm{H} \alpha$ line corroborate this picture as narrow fibrils with opposite line of sight motions located in immediate proximity to each other show up during the microflares. These Doppler shifted chromospheric fibrils, however, have a much lower line of sight velocity $\left(\sim 5 \mathrm{~km} \mathrm{~s}^{-1}\right)$ than in the CDS observations. Also, the fast change from redshifts to blueshifts observed at the northern footpoint of the third microflare could be due to twist motions.

Apart from the location of flare brightenings themselves, enhanced downflows in the chromosphere and transition region are also observed to the south of the strongest flare brightenings located in the penumbra of the sunspot. These flows reach velocities of $\sim+80 \mathrm{~km} \mathrm{~s}^{-1}$ which are much higher than the sound speed in the chromosphere which is on the order of $c_{\mathrm{s}} \approx 10 \mathrm{~km} \mathrm{~s}^{-1}$. Transient supersonic downflows in the photosphere and chromosphere are often observed at locations where new magnetic flux is formed or where moving magnetic features near sunspots are observed (e.g. recently Shimizu et al. 2008). Magnetogram sequences for the day also show motions of the magnetic flux at the microflares' site. Czaykowska et al. (1999) also reported downflows of $\sim+60 \mathrm{~km} \mathrm{~s}^{-1}$ into a sunspot during an M-class flare observed by CDS with no obvious origin of such flows. As a possible cause for the strong downflows into the penumbra associated with our microflares we propose solar gravitation. Indications of free falling plasma are usually observed in the late phase of flares when cooling flare plasma flows from the corona to deeper layers (see e.g. Brosius 2003; Del Zanna et al. 2006). For our microflares, we can speculate that in the course of the reconnection process, the magnetic field topology is reconfigured in such a way that material in the pre-reconnection magnetic configuration is not sustained any more and flows downwards in newly formed loops. Assuming the observed flows to be accelerated in free fall, we can estimate the height where they should originate. The solar gravitational acceleration is $g_{\odot} \approx 2.7 \times 10^{4} \mathrm{~cm} \mathrm{~s}^{-2}$ and the acceleration time for the 08:26 UT event was estimated to $t_{\text {acc }} \approx 120 \mathrm{~s}$ from the CDS velocity evolution in pixels showing strong downflows. With these parameters, the height where the plasma starts to accelerate can be back-extrapolated to $h \approx g_{\odot} \cdot t^{2} / 2 \approx 2 \times 10^{8} \mathrm{~cm}$. This is indeed still at a chromospheric level. Also, $h$ is comparable to the RHESSI loop height ${ }^{5}$ which we approximated to $h_{\mathrm{rh}} \approx 7 \times 10^{8} \mathrm{~cm}$. This could indicate a common formation process. So a free fall of plasma into the penumbra could in principle explain the CDS observations. Another possibility for the creation of enhanced downflows could be a structural change of a sunspot plume due to the microflare. When inspecting $\mathrm{O} \mathrm{V}$ and $\mathrm{Ne}$ VI raster scans obtained $\sim 90 \mathrm{~min}$ before the 08:26 UT microflare, a small sunspot plume is observed at the positon where during the microflares enhanced downflows are observed. In sunspot plumes, considerable downflows have been reported (see e.g. Maltby et al. 1999; Brynildsen et al. 2001; Brosius \& Landi 2005) and also for the sunspot plume in our raster images, downflows are observd $\left(\lesssim+40 \mathrm{~km} \mathrm{~s}^{-1}\right)$. Thus, it could be possible that the restructuring of the magnetic field in the microflare enhances the downflows in the plume.

\section{Conclusions}

Micro- and nanoflare coronal heating models require the occurrence of a multitude of reconnection events. Therefore it is important to evaluate whether small flares on the Sun behave like powerful flares, with the same physics underlying them and with the same processes occurring, hence the same creation mechanism, as regular flares. With our data set of $3 \mathrm{X}$-ray microflares emitting at the sensitivity threshold of current X-ray detectors, we are able to study many different aspects of the smallest X-ray microflares and the response of the solar atmosphere to these events.

In accordance with previous studies, our findings suggest that the analyzed microflares have multi-wavelength and magnetic field properties comparable to regular flares and behave consistently with events powered by magnetic reconnection in the corona. TRACE $17.1 \mathrm{~nm}$ imaging data for the first event show a complex appearance with several brightenings probably constituting the hot footpoints of the flaring loop system. Utilizing the DOT speckled data available for all three microflares, we were able to study aspects like their chromospheric

\footnotetext{
5 We estimated the RHESSI loop of the 08:26:30 UT event to be a half circle connecting the DOT $\mathrm{H} \alpha$ footpoint brightenings located at the end of the RHESSI 3-8 keV source.
} 
fine structure at a resolution of $0.2^{\prime \prime}-0.3^{\prime \prime}$ in unprecedented detail. The microflares were indeed found to be finely structured on the arcsecond level and associated small scale features like bright fibrils were discovered.

As regards the mass flow pattern associated with the events, the interpretation of results in the frame of chromospheric evaporation theory and hydrodynamic simulations is difficult. CDS observations of the three microflares indeed prove the existence of plasma flows in the chromosphere and transition region in the microflare brightenings. The flow direction and velocities suggest that explosive evaporation is occurring in the first and gentle evaporation in the second event. A more complex behavior is indicated for the third microflare. Observations from the coronal Si XII line and RHESSI spectroscopy, however, do not clearly support this picture. The electron energy flux densities estimated for two events showing non-thermal X-ray spectra are at the margin between gentle and explosive evaporation. Given the errors in measurements, it is impossible to determine whether the observed flows and electron fluxes in microflares are in agreement with the results proposed by simulations of beam driven evaporation. Details in the observed flow dynamics like short-time high velocity flows of opposite direction at the flare peaks indicative of twist motions reveal the complexity of the flare process even for such small events. CDS velocity data also show that dynamic processes are not only confined to the bright microflare site but also involve adjacent areas.

Acknowledgements. We would like to thank Ryan Milligan for his helpful comments. We also acknowledge the referee's contributions which improved the text. This work was supported by the exchange program SK-AT-0004-08 of the Österreichischer Austauschdienst ÖAD (Austria) and the Slovak Research and Development Agency SRDA (Slovakia) as well as by the SRDA project APVV0066-06. The University of Graz, the Austrian Academy of Sciences and the company L'Oréal Austria supported S. B.-S. with research scholarships. Data were acquired in the frame of the SOHO Joint Observing Program JOP 171 operated in cooperation with the TRACE satellite and the DOT. The authors are grateful for the cooperation of the SOHO, TRACE, and DOT teams during the run of JOP 171 in July 2006.

The DOT is operated by the Utrecht University at the Spanish Observatorio del Roque de los Muchachos of the Instituto de Astrofísica de Canarias. SOHO is a project of international cooperation between ESA and NASA. TRACE is a mission of the Stanford-Lockheed Institute for Space Research and part of the NASA Small Explorer program. RHESSI is a NASA small explorer mission. In the frame of this work, we used NASA's Astrophysics Data System.

\section{References}

Abbett, W. P., \& Hawley, S. L. 1999, ApJ, 521, 906

Acton, L. W., Finch, M. L., Gilbreth, C. W., et al. 1980, Sol. Phys., 65, 53

Allred, J. C., Hawley, S. L., Abbett, W. P., et al. 2005, ApJ, 630, 573

Antonucci, E., Gabriel, A. H., Acton, L. W., et al. 1982, Sol. Phys., 78, 107

Antonucci, E., Dodero, M. A., \& Martin, R. 1990, ApJS, 73, 147

Aschwanden, M. J. 2004, Phys. Solar Corona (Springer), 382

Aschwanden, M. J., Winebarger, A., Tsiklauri, D., et al. 2007, ApJ, 659, 1673
Brosius, J. W. 2003, ApJ, 586, 1417

Brosius, J. W., \& Holman, G. D. 2009, ApJ, 692, 492

Brosius, J. W., \& Landi, E. 2005, ApJ, 632, 1196

Brosius, J. W., \& Phillips, K. J. H. 2004, ApJ, 613, 580

Brynildsen, N., Maltby, P., Fredvik, T., Kjeldseth-Moe, O., \& Wilhelm, K. 2001,

Sol. Phys., 198, 89

Culhane, J. L., Bentley, R. D., Hiei, E., et al. 1991, Sol. Phys., 136, 89

Czaykowska, A., de Pontieu, B., Alexander, D., et al. 1999, ApJ, 521, L75

Del Zanna, G. D., Schmieder, B., Mason, H., Berlicki, A., \& Bradshaw, S. 2006, Sol. Phys., 239, 173

Delaboudinière, J.-P., Artzner, G. E., Brunaud, J., et al. 1995, Sol. Phys., 162, 291

Domingo, V., Fleck, B., \& Poland, A. I. 1995, Sol. Phys., 162, 1

Doschek, G. A., Feldman, U., Kreplin, R. W., et al. 1980, ApJ, 239, 725

Falchi, A., Teriaca, L., \& Maltagliati, L. 2006, Sol. Phys., 239, 193

Feldman, U., Doschek, G. A., Kreplin, R. W., et al. 1980, ApJ, 241, 1175

Fisher, G. H. 1989, ApJ, 346, 1019

Fisher, G. H., Canfield, R. C., \& McClymont, A. N. 1985a, ApJ, 289, 434

Fisher, G. H., Canfield, R. C., \& McClymont, A. N. 1985b, ApJ, 289, 425

Fisher, G. H., Canfield, R. C., \& McClymont, A. N. 1985c, ApJ, 289, 414

Fludra, A., Bentley, R. D., Lemen, J. R., Jakimiec, J., \& Sylwester, J. 1989, ApJ, 344, 991

Gömöry, P., Rybák, J., Kučera, A., Curdt, W., \& Wöhl, H. 2006, A\&A, 448, 1169

Hammerschlag, R. H., \& Bettonvil, F. C. M. 1998, New Astron. Rev., 42, 485

Handy, B. N., Acton, L. W., Kankelborg, C. C., et al. 1999, Sol. Phys., 187, 229

Hannah, I. G., Christe, S., Krucker, S., et al. 2008, ApJ, 677, 704

Harrison, R. A., Sawyer, E. C., Carter, M. K., et al. 1995, Sol. Phys., 162, 233

Hurford, G. J., Schmahl, E. J., Schwartz, R. A., et al. 2002, Sol. Phys., 210, 61

Kamio, S., Kurokawa, H., Brooks, D. H., Kitai, R., \& UeNo, S. 2005, ApJ, 625, 1027

Krucker, S., Christe, S., Lin, R. P., Hurford, G. J., \& Schwartz, R. A. 2002, Sol. Phys., 210, 445

Kundu, M. R., Schmahl, E. J., Grigis, P. C., Garaimov, V. I., \& Shibasaki, K. 2006, A\&A, 451, 691

Lin, R. P., Dennis, B. R., Hurford, G. J., et al. 2002, Sol. Phys., 210, 3

Liu, C., Qiu, J., Gary, D. E., Krucker, S., \& Wang, H. 2004, ApJ, 604, 442

MacPherson, K. P., \& Jordan, C. 1999, MNRAS, 308, 510

Maltby, P., Brynildsen, N., Fredvik, T., Kjeldseth-Moe, O., \& Wilhelm, K. 1999, Sol. Phys., 190, 437

Mariska, J. T., Doschek, G. A., \& Bentley, R. D. 1993, ApJ, 419, 418

Milligan, R. O. 2008, ApJ, 680, L157

Milligan, R. O., Gallagher, P. T., Mathioudakis, M., et al. 2006, ApJ, 638, L117

Parker, E. N. 1988, ApJ, 330, 474

Peter, H., \& Judge, P. G. 1999, ApJ, 522, 1148

Priest, E. R., \& Forbes, T. G. 2002, A\&A Rev., 10, 313

Qiu, J., Liu, C., Gary, D. E., Nita, G. M., \& Wang, H. 2004, ApJ, 612, 530

Rutten, R. J., Hammerschlag, R. H., Bettonvil, F. C. M., Sütterlin, P., \& de Wijn, A. G. 2004, A\&A, 413, 1183

Scherrer, P. H., Bogart, R. S., Bush, R. I., et al. 1995, Sol. Phys., 162, 129

Schwartz, R. A., Csillaghy, A., Tolbert, A. K., et al. 2002, Sol. Phys., 210, 165

Shimizu, T., Lites, B. W., Katsukawa, Y., et al. 2008, ApJ, 680, 1467

Smith, D. M., Lin, R. P., Turin, P., et al. 2002, Sol. Phys., 210, 33

Stoiser, S., Veronig, A. M., Aurass, H., et al. 2007, Sol. Phys., 246, 339

Stoiser, S., Brown, J. C., \& Veronig, A. M. 2008, Sol. Phys., 250, 315

Sturrock, P. A. 1973, NASA Spec. Publ., 342, 3

Sütterlin, P., Hammerschlag, R. H., Bettonvil, F. C. M., et al. 2001, in Advanced Solar Polarimetry - Theory, Observation, and Instrumentation, ed. M. Sigwarth, ASP Conf. Ser., 236, 431

Teriaca, L., Falchi, A., Falciani, R., Cauzzi, G., \& Maltagliati, L. 2006, A\&A, 455,1123 


\section{Appendix A: Movie description}

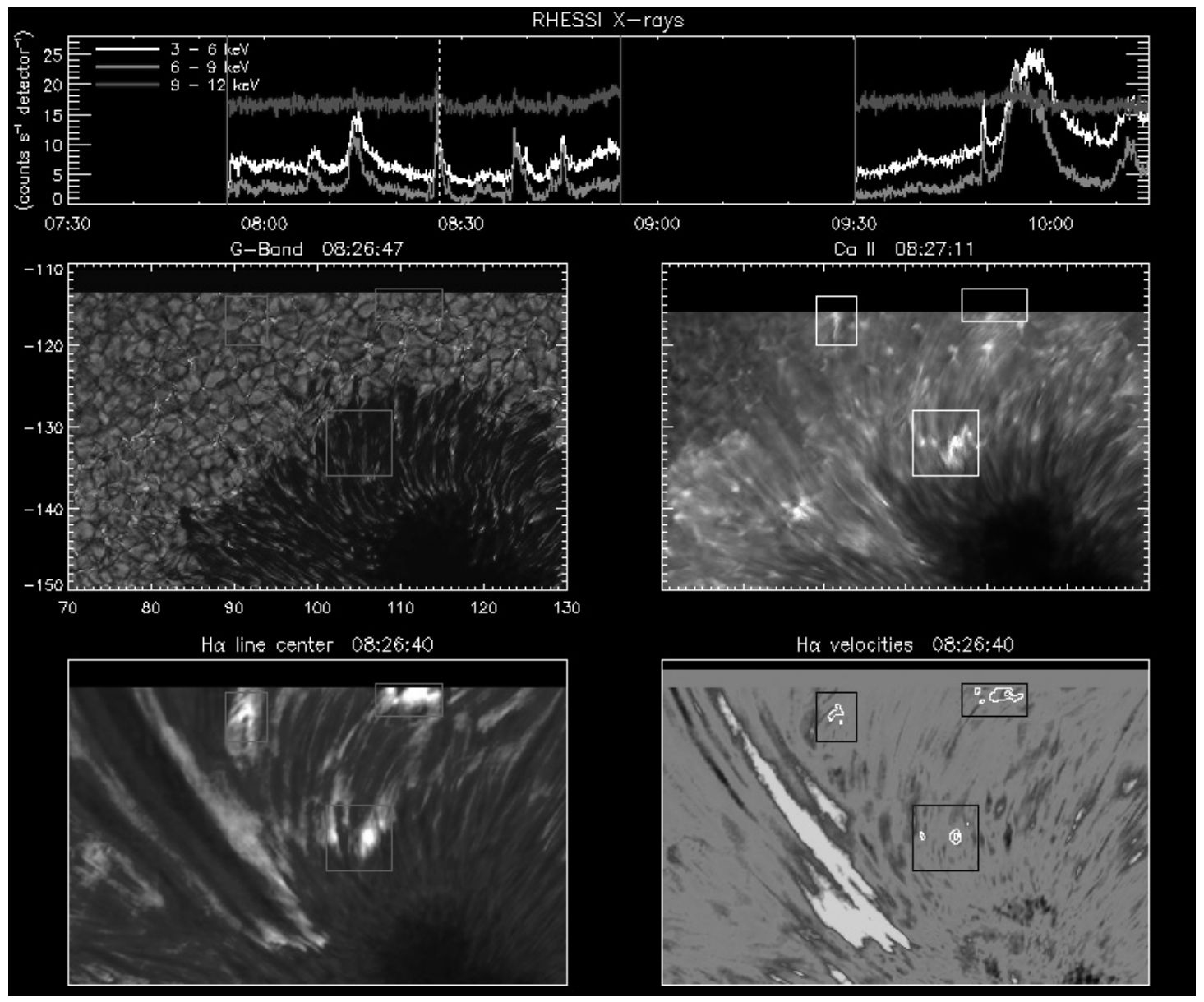

Fig. A.1. Black and white snapshot of a movie showing photospheric and chromospheric images obtained during the DOT observing run on July 4, 2006 ( 07:45 UT to 10:09 UT). The movie (in color) is provided online as supplementary material to this paper. The DOT data are combined with the RHESSI light curves in the energy bands 3-6 keV (white), 6-9 keV (green) and 9-12 keV. Data gaps are due to RHESSI night times. The three microflares presented in this work peaked at 08:26 UT, 08:38 UT and 08:45 UT. The vertical white dashed line sliding through the RHESSI light curve plot marks the recording time of the DOT filtergrams and dopplergrams shown in the 4 bottom panels (G Band, Ca II H, H $\alpha$ intensities and velocities). The $\mathrm{H} \alpha$ line of sight dopplergram is scaled to $[-5,+5] \mathrm{km} \mathrm{s}^{-1}$ where upflows appear in blue and black and downflows in red and yellow. White contours in the dopplergrams enclose emission $>70 \%$ and $90 \%$ of the maximum intensity observed in the $\mathrm{H} \alpha$ line center. The frame boxes in each DOT image point out the location of enhanced chromospheric emission in the three microevents. The northern edge of the DOT images varies for the filters and for different times due to the speckle reconstruction process. 